ARTICLE

https://doi.org/10.1038/s41467-019-12336-w

\title{
Damage sensing by a Nox-Ask1-MKK3-p38 signaling pathway mediates regeneration in the adult Drosophila midgut
}

Parthive H. Patel (1) 1,2,3,4,5, Clothilde Pénalva ${ }^{3,6}$, Michael Kardorff5,6, Marianne Roca5,6, Bojana Pavlović5,6, Anja Thiel ${ }^{5}$, Aurelio A. Teleman (10 ${ }^{4} \&$ Bruce A. Edgar ${ }^{3}$

Epithelia are exposed to diverse types of stress and damage from pathogens and the environment, and respond by regenerating. Yet, the proximal mechanisms that sense epithelial damage remain poorly understood. Here we report that p38 signaling is activated in adult Drosophila midgut enterocytes in response to diverse stresses including pathogenic bacterial infection and chemical and mechanical insult. Two upstream kinases, Ask 1 and Licorne (MKK3), are required for p38 activation following infection, oxidative stress, detergent exposure and wounding. Ask1-p38 signaling in enterocytes is required upon infection to promote full intestinal stem cell (ISC) activation and regeneration, partly through Upd3/JakStat signaling. Furthermore, reactive oxygen species (ROS) produced by the NADPH oxidase Nox in enterocytes, are required for p38 activation in enterocytes following infection or wounding, and for ISC activation upon infection or detergent exposure. We propose that NoxROS-Ask1-MKK3-p38 signaling in enterocytes integrates multiple different stresses to induce regeneration.

\footnotetext{
${ }^{1}$ Elizabeth Blackwell Institute for Health Research, University of Bristol, Bristol BS8 1UH, UK. ${ }^{2}$ School of Cellular and Molecular Medicine, University of Bristol, Bristol BS8 1TD, UK. ${ }^{3}$ Huntsman Cancer Institute, University of Utah, Salt Lake City, UT 84112, USA. ${ }^{4}$ German Cancer Research Center (DKFZ), 69120 Heidelberg, Germany. ${ }^{5}$ Center for Molecular Biology, University of Heidelberg (ZMBH), 69120 Heidelberg, Germany. ${ }^{6}$ These authors contributed equally: Clothilde Pénalva, Michael Kardorff, Marianne Roca, Bojana Pavlović. Correspondence and requests for materials should be addressed to P.H.P. (email: p.patel@bristol.ac.uk) or to B.A.E. (email: bruce.edgar@hci.utah.edu)
} 
pithelial tissues are regularly exposed to a variety of stresses caused by pathogens, toxins, and other environmental factors. Nevertheless, barrier epithelia have an incredible ability to not only defend themselves from these insults but also to repair themselves. One exquisite example is the intestinal epithelium, which upon infection (1) defends itself against pathogens by producing mucus, reactive oxygen species (ROS), and antimicrobial peptides (AMPs), and (2) mobilizes resident intestinal stem cells (ISCs) to rapidly replace lost epithelial cells to maintain epithelial integrity ${ }^{1}$. While epithelia have sophisticated systems to sense tissue stress and promote defense responses, how stress sensing is achieved and then coupled to epithelial regeneration is not well understood.

Similar to the mammalian intestine, the adult Drosophila intestine (or midgut) is maintained by ISCs, which produce transient progeny, enteroblasts (EBs) that can differentiate into either enterocytes (ECs) or enteroendocrine cells (EEs) ${ }^{2,3}$. These epithelial cells grow upon a basement membrane (BM) overlaying the visceral muscle (VM), which together with the EBs, ECs, and EEs comprise the epithelial ISC niche ${ }^{4}$. Upon damage, intestinal regeneration in Drosophila is mediated by the cytokine/JAKSTAT, EGFR/Ras/MAPK, insulin, TOR, Wnt/Wg, BMP/TGF $\beta$, PDGF/VEGF, Hh, Notch, JNK), Hpo, and Ret signaling ${ }^{5,6}$. The various niche cells (EBs, ECs, and VM) together provide most of these signals to regulate ISC proliferation, self-renewal, and differentiation ${ }^{5}$. Furthermore, the Drosophila ISC niche can sense pathogenic, chemical, and mechanical stresses such as Erwinia carotovora carotovora (Ecc15), Pseudomonas entomophila (P.e.), ROS (paraquat), detergents (dextran sodium sulfate, DSS), DNA damage (bleomycin), tumor growth, and EC detachment from the BM through the JNK and Hpo signaling pathways ${ }^{7-12}$. However, precisely how these pathways sense these diverse types of stresses and couple them to tissue regeneration is still not well understood.

Stress-activated protein kinases (SAPKs), which include the Jun-N-terminal kinase (JNK) and the p38 mitogen-activated protein kinase (MAPK), are a conserved subfamily of MAPKs that sense and elicit cellular responses to stress ${ }^{13}$. SAPKs are thought to promote both fly intestinal regeneration and immunity. JNK is activated in ECs by stress (i.e., ROS (paraquat), pathogenic bacterial infection $(E c c 15$, P.e.), tumor growth, and detachment from the BM), and increasing JNK activity in ECs is sufficient to promote ISC proliferation by stimulating cytokine (upd2, upd3) expression ${ }^{8-10,14}$. These cytokines facilitate regeneration by stimulating JAK-STAT activity in ISCs to promote their proliferation and in EBs to promote their differentiation $8,10,15-17$. Together, these data have led to the common view that JNK signaling can sense stress and couple it to intestinal regeneration ${ }^{18}$. Nevertheless, JNK was found to be only mildly required in ECs for resistance (or survival) to Ecc $15^{10}$ and for upd 3 induction upon $E c c 15$ infection $^{19}$, suggesting that other pathways in addition to JNK are required to couple stress to regeneration.

On the other hand, previous work in Caenorhabditis elegans, the adult fly intestine, and human cultured intestinal cancer cells suggest that p38 signaling is required for sensing and providing resistance toward pathogenic bacteria ${ }^{20,21}$. It has been reported in the fly intestine that bacterial wall components (i.e., peptidoglycans) and metabolites (i.e., uracil) activate p38 signaling, which subsequently confers resistance by boosting ROS generation (i.e., $\mathrm{H}_{2} \mathrm{O}_{2}$ ) by upregulating the expression of the nicotinimide adenine dinucleotide phosphate (NADPH) oxidase (Nox) and Dual oxidase (Duox) ${ }^{21,22}$. Indeed, activated p38a, one of the three fly p38 MAPKs (p38a, p38b, and p38c), was shown to upregulate Duox expression through phosphorylation and activation of the activating transcription factor 2 (Atf2) $)^{21,22}$. In contrast, another
Drosophila study found that $\mathrm{p} 38 \mathrm{a}$ and $\mathrm{p} 38 \mathrm{~b}$ mutant flies were as sensitive to P.e. infection as control flies, and that $\mathrm{p} 38$ a was not required for Duox expression upon infection ${ }^{23}$. Instead, they reported that $\mathrm{p} 38 \mathrm{c}$ is upregulated after pathogenic bacterial infection to not only promote Duox-mediated ROS production via Atf2 but also to restrict ISC proliferation by limiting cytokine (Upd3) expression ${ }^{23}$. Although it is not clear which fly p38 (p38a or $\mathrm{p} 38 \mathrm{c}$ ) is involved, these data suggest that p38 signaling promotes fly intestinal immunity through Duox-mediated ROS production. However, whether p38 signaling plays an important role in Drosophila intestinal regeneration is not known.

Work in the mouse intestinal epithelium suggests a role for p38 signaling in intestinal regeneration; $p 38 \alpha$ (one of four p38s in mice and homologous to fly $\mathrm{p} 38 \mathrm{a}$ and $\mathrm{p} 38 \mathrm{~b}$ ) was required in the mouse intestinal epithelium for repair after detergent (DSS)induced damage ${ }^{24,25}$. Consistent with this, p38b signaling in fly ECs was also shown to partially regulate upd3 expression in Ecc15-infected flies ${ }^{19}$; however, its requirement for ISC response and midgut regeneration was not directly tested. Although these studies suggest a role for p38 in intestinal regeneration, how p38 is activated upon intestinal damage, which cell types utilize it, and how p38 promotes intestinal regeneration are not well understood.

ROS are also thought to promote intestinal turnover and regeneration. The NADPH oxidase, Nox, has been reported to produce ROS $\left(\mathrm{O}_{2}{ }^{\bullet}\right)$ upon exposure to commensal bacteria such as Lactobacillus plantarum, which also consequently promotes fly and mouse ISC proliferation ${ }^{26}$. Furthermore, paraquat or $\mathrm{H}_{2} \mathrm{O}_{2}$ exposure promote fly intestinal epithelial turnover ${ }^{10}$. In contrast, antioxidants (i.e., $\mathrm{N}$-acetyl cysteine and glutathione) feeding or Duox depletion blocked intestinal regeneration in Ecc15-infected flies $^{10}$. Moreover, Nox1 knockout mice showed reduced ISC proliferation after DSS exposure ${ }^{27}$. Although ROS can stimulate JNK activity ${ }^{14}$, how ROS promote intestinal regeneration is not well understood. In addition, although it has been reported that intestinal ROS production is boosted by p38 signaling, it is not known whether ROS can activate p38 signaling to effect intestinal regeneration.

SAPKs (JNK, p38) are activated by the upstream SAP kinase kinases (SAP2Ks) such as MKK4/7 and MKK3/6, which activate JNK and p38, respectively ${ }^{13}$. SAP2Ks such as MKK3 are dualspecificity kinases that can phosphorylate the highly conserved dual-phosphorylation site (TGY) of p38s to further promote their activity $^{13,28}$. The sole fly MKK3, Licorne, can phosphorylate both p38a and p38b but not p38c, which lacks the dualphosphorylation $\operatorname{sit}^{29}$. SAP2Ks are activated by SAP kinase kinase kinases (SAP3Ks) (i.e., MAPK/ERK kinase kinase 1 $(\mathrm{Mekk} 1)^{30}$, Apoptosis signal-regulating kinase 1 (Ask1), transforming growth factor- $\beta$ (TGF $\beta$ )-activated kinase 1 (Tak1)), which are activated by a variety of stresses, such as heat, oxidative, osmotic, or pathogen. In flies, both Mekk1 and Tak1 signaling have been reported to play important roles in sensing pathogenic bacteria and promoting gut immunity $21,23,31$. In C. elegans, Ask1/ nsy-1 is required for pathogen resistance ${ }^{20}$. Ask 1 is also thought to sense oxidative stress $^{32}$ and in flies it promotes wing disc regeneration via JNK and $\mathrm{p} 38^{33}$. A role for SAP2Ks and SAP3Ks in intestinal regeneration, however, is unknown. As ROS promote tissue regeneration in other contexts ${ }^{34-36}$, SAP3Ks such as Ask1 may have an important role in coupling intestinal immunity to regeneration.

Here, we show that p38 signaling promotes intestinal regeneration by sensing a variety of stresses in ECs and promoting ISC proliferation. In addition, we find that Nox-derived ROS and Ask1 are required to fully activate MKK3-p38 signaling to promote intestinal regeneration upon pathogenic infection. Furthermore, we find that Nox-derived ROS are required to promote 

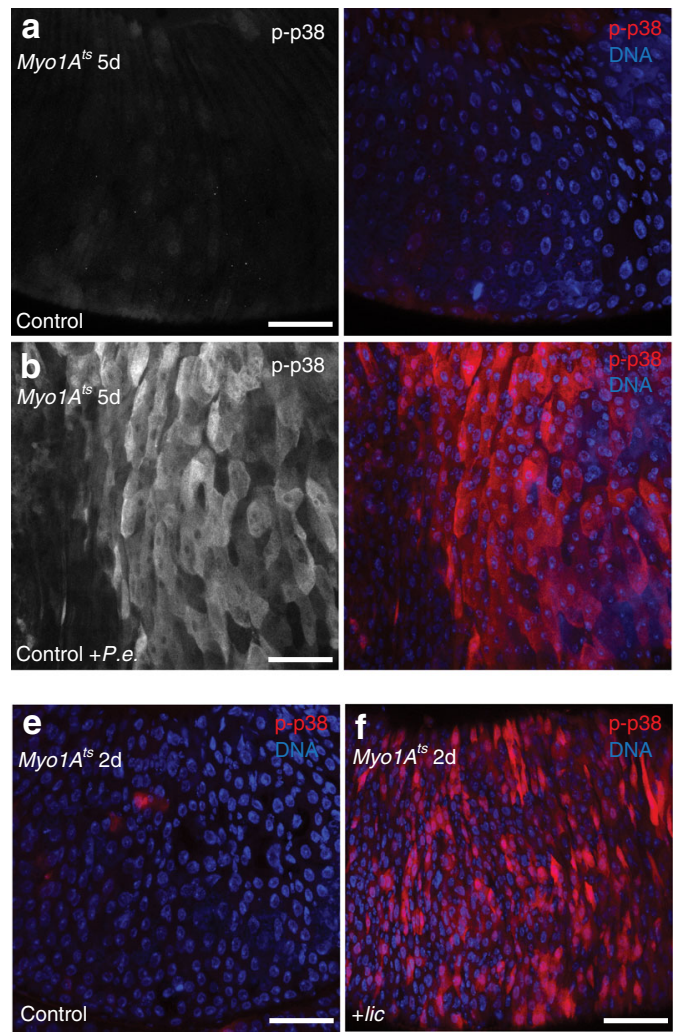
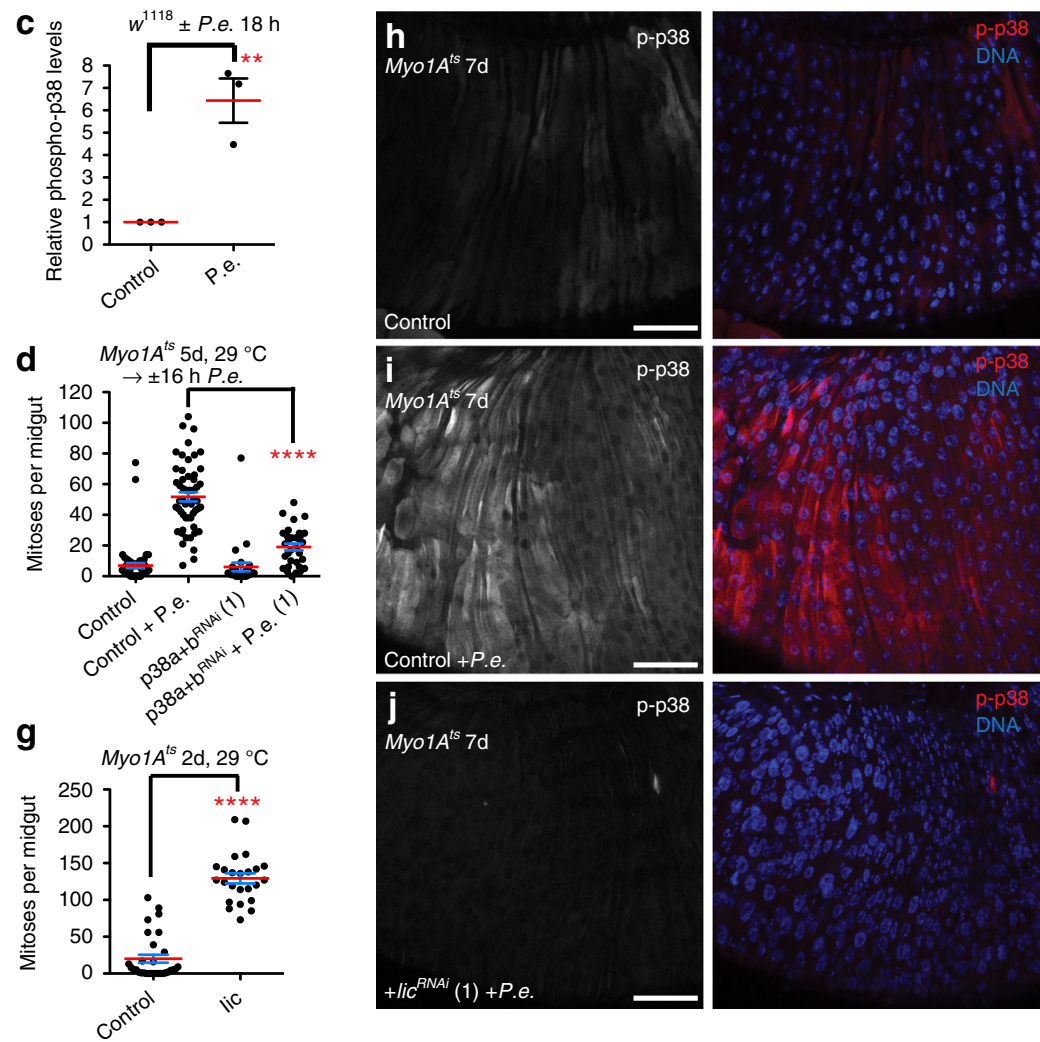

Fig. 1 Mkk3-p38 signaling in ECs promotes ISC proliferation upon damage. a, b Both p38a and p38b (p38a + b) are activated in ECs upon P.e. infection. p38 activation increased in ECs of P.e.-infected control (EC-specific, Myo1A ${ }^{\text {ts}}$ ) midguts (b right, red) compared with uninfected control midguts (a right, red). c p38 is activated in the midguts upon pathogen infection. Mean (with s.e.m.) phospho-p38 levels are increased in P.e.-infected midguts ( $n=3$ midguts from one experiment) compared with uninfected control midguts ( $n=3$ midguts from one experiment) (unpaired $t$ test (on data not normalized to control); $P=0.0085$ ). $\mathbf{d}$ Both $\mathrm{p} 38 \mathrm{a}$ and $\mathrm{p} 38 \mathrm{~b}$ are required in ECs to promote ISC proliferation upon P.e. infection. The ISC response is blocked in $P$.e.infected midguts expressing $p 38 a+b^{R N A i}$ in ECs for 5 days with Myo1 $A^{\text {ts }}$ compared with P.e.-infected control (Myo1A ${ }^{\text {ts }}$ ) midguts (Mann-Whitney; $P<$ 0.0001). The mean number of phosphorylated histone H3 Ser 10-positive cells per midgut with s.e.m. in control midguts ( $n=55$ midguts), P.e.-infected control midguts ( $n=53$ midguts), midguts expressing $p 38 a+b^{R N A i}$ in ECs for 5 days ( $n=28$ midguts) and P.e.-infected midguts expressing p38a $+b^{R N A i}$ in ECs for 5 days ( $n=32$ midguts). Midguts pooled from three independent experiments. e, $\mathbf{f}$ Mkk3/Licorne can activate p38 in ECs. p38 activation increased in the midguts overexpressing Licorne in ECs for 2 days with Myo1A ${ }^{\text {ts }}$ (f, red) compared with control midguts (e red). $\mathbf{g}$ Overexpression of Mkk3/ Licorne in ECs for 2 days with Myo1A ts promotes ISC proliferation. The mean number of phosphorylated histone H3 Ser 10 -positive cells per midgut with s. e.m. in control (Myo1A ${ }^{\text {ts }}$ ) midguts ( $n=31$ midguts) and in midguts expressing lic in ECs for 2 days with Myo1 $A^{\text {ts }}$ ( $n=24$ midguts) (Mann-Whitney; $p<$ 0.0001). Midguts pooled from three independent experiments. $\mathbf{h}-\mathbf{j}$ Mkk3/Licorne is required for p38 activation in ECs upon P.e. infection. p38 activation increases in ECs in P.e.-infected control (Myo1Ats) midguts (i right, red) compared with uninfected control midguts (h right, red). p38 activation is blocked however in P.e.-infected midguts expressing lic ${ }^{R N A i}$ for 7 days with Myo1A ${ }^{\text {ts }}$ (j right, red) compared with P.e.-infected control midguts. DNA is in blue. Scale bars in $\mathbf{a}, \mathbf{b}, \mathbf{e}, \mathbf{f}, \mathbf{h}-\mathbf{j}$ are $50 \mu \mathrm{m}$. Representative images in $\mathbf{a}, \mathbf{b}, \mathbf{e}, \mathbf{f}$ from three experiments; $\mathbf{h}$-j, two experiments. Source data are provided as a Source Data File

intestinal regeneration upon detergent exposure. Together, our data suggest that Nox-Ask1-MKK3-p38 signaling senses multiple different stresses and effectively couples these stresses with regeneration.

\section{Results}

p38 in ECs promotes ISC-mediated regeneration upon damage. Previously, p38 was described to be mostly inactive in the adult fly midgut $^{21}$. Using an anti-phospho-p38 antibody that should recognize both activated $\mathrm{p} 38 \mathrm{a}$ and $\mathrm{p} 38 \mathrm{~b}$, we found that in both male and female midguts p38 was activated often in the anterior midgut (R1, anterior R2) and occasionally in (and around) the mid-midgut $(\mathrm{R} 3)^{37}$. In the posterior midgut (R4a-R5), p38 activity was typically absent and we only occasionally observed low levels of activated p38. p38a was shown to be activated in midgut ECs after feeding of soluble microbial extract or peptidoglycan from $E c c 15^{21}$. Consistent with this result, we found that p38 was strongly activated in ECs throughout the midgut after oral infection with another Gram-negative bacterium P.e. relative to uninfected control midguts (Fig. 1a-c). Single depletion of either $\mathrm{p} 38 \mathrm{a}$ or $\mathrm{p} 38 \mathrm{~b}$ in ECs with UAS-RNA $i$ transgenes under the control of the EC-specific driver Myo1A-GAL4 $\left(M y o 1 A^{t s}\right)$ reduced neither the basal active p38 levels nor the p38 activation after P.e. infection relative to controls (Supplementary Fig. 2a-d). Although depletion of both p38a and p38b simultaneously $(p 38 a+b)$ using an $R N A i$ that targets both mRNAs with $M y o 1 A^{\text {ts }}$ did not detectably reduce the basal levels of p38 activation, it did however suppress the p38 activation upon P.e. infection relative to control-infected midguts (Supplementary Fig. 1a, b). This suggested that the phospho-p38-specific antibody recognizes both the phosphorylatable forms of fly p38 (p38a and p38b) but not p38c, which lacks the phosphorylation site 29,38 . Infection by pathogenic bacteria stimulates ISC proliferation and 
the replacement of stressed or dying $\mathrm{ECs}^{8,10}$. To determine whether p38 activation after P.e. infection occurred in newborn or old ECs, or both, we labeled ISCs and their progeny with green fluorescent protein (GFP) using the ISC lineage tracing tool esgGAL4 tubGAL80 ${ }^{\text {ts }}$ UAS-GFP; UAS-FLP, act $<C D C>$ GAL4 $\left(e s g^{\text {ts }} F / O\right)^{8}$. During P.e. infection, p38 activation was observed in both newborn and old ECs in the epithelium, but at low levels in ECs of controls (Supplementary Fig. 1c, d). These data suggest that both old and new ECs can sense stress during pathogenic bacterial infection and activate $\mathrm{p} 38 \mathrm{a}+\mathrm{b}$ signaling.

In addition to ECs, we observed strong p38 activation in the VM after P.e. infection relative to the VM of control, uninfected midguts (Supplementary Fig. 1e-f). To determine whether p38 activity in ECs and the VM was important for the ISC response to infection, we depleted both $p 38 a+b$ in the VM with how-GAL4, tubGAL80 ${ }^{t s}\left(\right.$ how $\left.^{t s}\right)$ or in ECs with Myo1 $A^{\text {ts }}$. Depletion of $p 38 a+$ $b$ in VM neither affected basal ISC divisions relative to uninfected control midguts nor the ISC response to P.e. infection relative to infected control midguts (Supplementary Fig. 1g). Although depletion of $p 38 a, p 38 b$, or both $p 38 a+b$ or overexpression of $\mathrm{p} 38^{\mathrm{DN}}$ in ECs did not affect basal ISC divisions relative to uninfected control midguts, blocking both $p 38 a+b$ in ECs, but neither $p 38 a$ or $p 38 b$ alone, did strongly suppress (by $63.2 \%$ with RNAi (1); 57.9\%, RNAi (2)) the ISC response to P.e. infection relative to infected controls (Fig. 1d; Supplementary Figs. 1h, j and $2 \mathrm{e}-\mathrm{g}$ ). Interestingly, we found that the ISC response to P.e. infection was mildly suppressed in the midguts depleted for $p 38 b$ in ECs (by $24.1 \%$ ) or mutant for $p 38 b$ ( $\left.p 38 b^{\text {ex9 }}\right)(22.5 \%$ in $\left.p 38 b^{e x 9} /+; 37.2 \%, p 38 b^{e x 9} / p 38 b^{e x 9}\right)^{39}$ (Supplementary Fig. $2 g, i$ ). In contrast, the ISC response in $p 38 a$ and $p 38 c$ double-mutant $\left(p 38 a^{1}\right)$ midguts ${ }^{23,29}$ was not blocked upon P.e. infection (Supplementary Fig. 2h). Together, these data suggest that upon P.e. infection, $p 38 b$ is required in ECs for the ISC response, and that $p 38 a$ may be required to sense stress in the absence of $p 38 b$ or together with $p 38 b$. These data suggest that $\mathrm{p} 38 \mathrm{a}+\mathrm{b}$ signaling is required in ECs to promote ISC-mediated regeneration.

The SAP2K, MKK3, is known to activate p38 in mammalian cells $^{13,28}$; however, its ability to activate p38 in intestinal epithelial cells is not known. Moreover, stress responses known to be p38dependent (i.e., osmotic stress in the larval Drosophila hindgut) have been previously shown to not require MKK $3^{40}$. We found that overexpression of the Drosophila MKK3, licorne (lic), with the Myo1 ${ }^{t s}$ driver strongly activated p38 in ECs (Fig. 1e, f; Supplementary Fig. $3 \mathrm{a}-\mathrm{c}$ ). Furthermore, Lic overexpression in ECs for 2 days also increased ISC proliferation relative to basal ISC divisions in control midguts (Fig. 1g). Conversely, we found that while depletion of the fly MKK3, licorne, in ECs with Myo1 ${ }^{\text {ts }}$ did not noticeably affect basal active p38 levels relative to control uninfected midguts, it inhibited p38 activation upon P.e. infection (Fig. 1h-j, red; Supplementary Fig. $3 \mathrm{~d}-\mathrm{i}$ ). These data suggest that $\mathrm{p} 38$ is activated upon infection via MKK3/Lic resulting in ISC proliferation.

The boost in ISC proliferation after P.e. infection is partly due to increased mitogen production (i.e., cytokines: $\mathrm{Upd} 2, \mathrm{Upd} 3$ ) by $\mathrm{ECs}^{8}$. Indeed, we found that mRNA levels encoding Upd 3 and the JAK-STAT target, Socs36E, were increased after a variety of stresses, including P.e., Ecc15, $\mathrm{H}_{2} \mathrm{O}_{2}$, Sodium dodecyl sulfate (SDS), bleomycin, and heat shock (Fig. 2a). To determine the role of p38 in this stress-dependent cytokine induction, we tested whether upd3 mRNA was induced after P.e. infection in p38depleted midguts. Using quantitative PCR (qPCR), we found that EC-targeted RNAis against $p 38 b$ and $p 38 a+b$ suppressed the induction of $u p d 3$ mRNA following P.e. infection by 62 and $49 \%$, respectively (Fig. 2b). In support of a role for $\mathrm{p} 38 \mathrm{a}+\mathrm{b}$ signaling in regulating cytokine production, Licorne overexpression for 24 $\mathrm{h}$ was sufficient to induce $u p d 2$ and $u p d 3$ expression $(\approx 8$-fold $)$
(Fig. 2c). These data suggest that MKK3-p38 signaling promotes upd3 induction upon P.e. infection, largely through p38b. Cytokine-induced JAK-STAT activity in the midgut can be detected using the in vivo reporter, 10x STAT-GFP, which we found to be high in progenitors and a few ECs in uninfected controls and in uninfected controls depleted for $p 38 a+b$ (Fig. 2d; Supplementary Fig. 1i). However, P.e.-infected controls showed strongly increased 10x STAT-GFP in most ECs, whereas this was markedly decreased in $p 38 a+b$-depleted midguts (Fig. 2e-g). These data suggest that p38 signaling in ECs promotes ISCmediated regeneration by boosting cytokine Upd3 expression.

We also tested if known p38 phosphorylation targets were required in ECs to promote ISC response to infection by assessing whether ISC proliferation was affected in P.e.-infected flies mutant for the transcriptional regulator Atf2 $\left(A t f 2^{P B}\right)^{41}$ or in flies depleted for Atf2 in ECs, or mutant for MAPK-activated protein kinase 2 (MAPKAP-K2 or MK2) ${ }^{40}$. We found that basal ISC division rates were not affected in Atf2 mutant midguts, or in midguts depleted of Atf2 in ECs (Supplementary Fig. 4a, b, d). Furthermore, we did not find an effect on the ISC response to P.e. infection in Atf2 mutant midguts, or in midguts depleted for Atf2 in ECs (Supplementary Fig. 4a, b). Similarly, the ISC response to P.e. infection was not affected in the MK2 P-element excision deletion mutant $\left(M K 2^{\Delta 43}\right)$ when compared with its precise excision control, $M K 2^{\Delta 1 A}$ (Supplementary Fig. 4c). These data suggest that p38 activity in stressed ECs does not promote ISC proliferation via MK2 or ATF2.

Chemical and mechanical stress activate p38 in the midgut. To test whether stresses in addition to pathogenic bacteria can activate $\mathrm{p} 38$, we tested whether several other types of stress could activate p38 in midgut ECs. Exposing flies or fly cultured S2 cells to a diverse array of stresses (i.e., Gram-negative pathogenic bacteria (Ecc15, P.e.), septic injury (Salmonella), heat shock, dry starvation, ROS (i.e., hydrogen peroxide $\left(\mathrm{H}_{2} \mathrm{O}_{2}\right)$, high salt $(0.2 \mathrm{M}$ $\mathrm{NaCl}$ ), metal ions) has been shown to activate p38 $21,29,38$; however, only enteric infection had been shown to activate p38 in the adult fly midgut ${ }^{21}$. We found that $\mathrm{p} 38$ signaling was not activated in midguts after heat shock at $37^{\circ} \mathrm{C}$ for $30 \mathrm{~min}$ (Supplementary Fig. 5a, b). However, in vivo reporters for JNK activity (puc-lacZ) and cytokine upd3 expression (upd3-lacZ) were activated after heat stress (Supplementary Fig. 5c-f). We also found that feeding $0.2 \mathrm{M} \mathrm{NaCl}$ or $0.4 \mathrm{M} \mathrm{NaCl}$ for 1 week (Supplementary Fig. $5 \mathrm{~g}, \mathrm{~h}$ ) or starvation (water only) did not activate p38 in midguts. These data suggest that heat, high salt stress, or starvation stress were not able to activate p38 signaling in the fly midgut.

We next tested mechanical stress. Strong p38 activation was observed in the VM and ECs shortly after wounding the posterior midgut with a needle (Fig. 3a, b; Supplementary Fig. 9a, b). Interestingly, p38 was activated not only near the wound site but also in the VM (and in many ECs) throughout the midgut, suggesting propagation of stress signaling (Fig. 3b; Supplementary Fig. 9b). Previously, we reported that EC detachment by integrin subunit depletion or by rapidly growing, differentiation-defective ISC tumors activates JNK and YAP/Yki activity ${ }^{9}$. Similarly, we found that ISC tumor growth ${ }^{9}$ also activated p38 in surrounding ECs relative to control midguts bearing no tumors (Fig. 3c, d). Moreover, detaching ECs by depleting the integrin subunit $\beta$ PS1 (myospheroid) or both aPS3 (scab) + aPS4 activated p38 signaling (Fig. 3e, f). Lastly, feeding flies with $1 \% \mathrm{H}_{2} \mathrm{O}_{2}$ or $0.2 \%$ sodium dodecyl sulfate (SDS) for 2 days activated p38 in the midgut relative to midguts from flies fed water alone (Fig. $4 a, b, d$, e). Together these data suggest that p38 signaling responds to several different stresses, including pathogenic infection, chemical stress, and mechanical stress. 
a

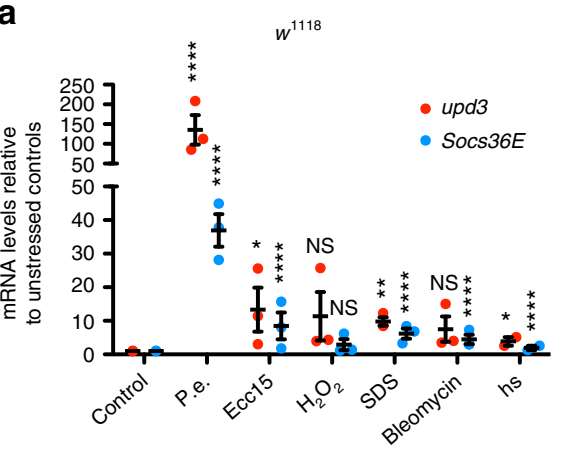

b

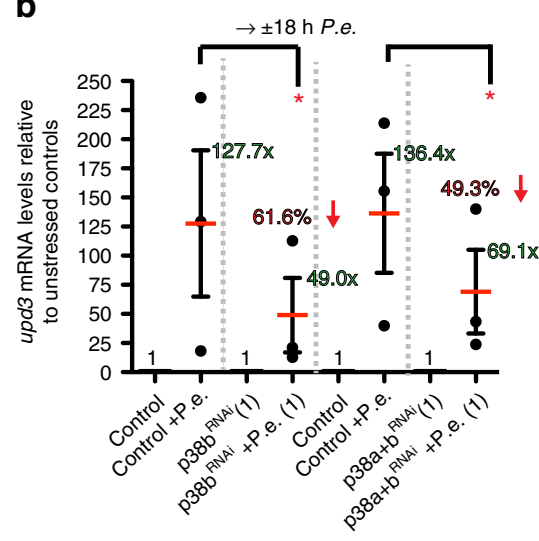

C

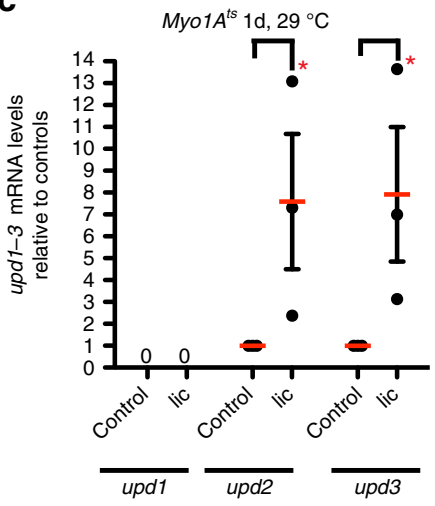

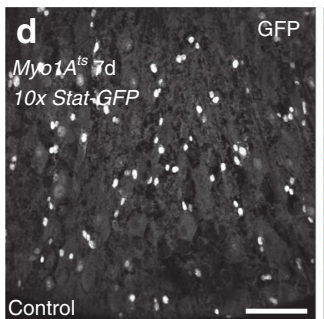
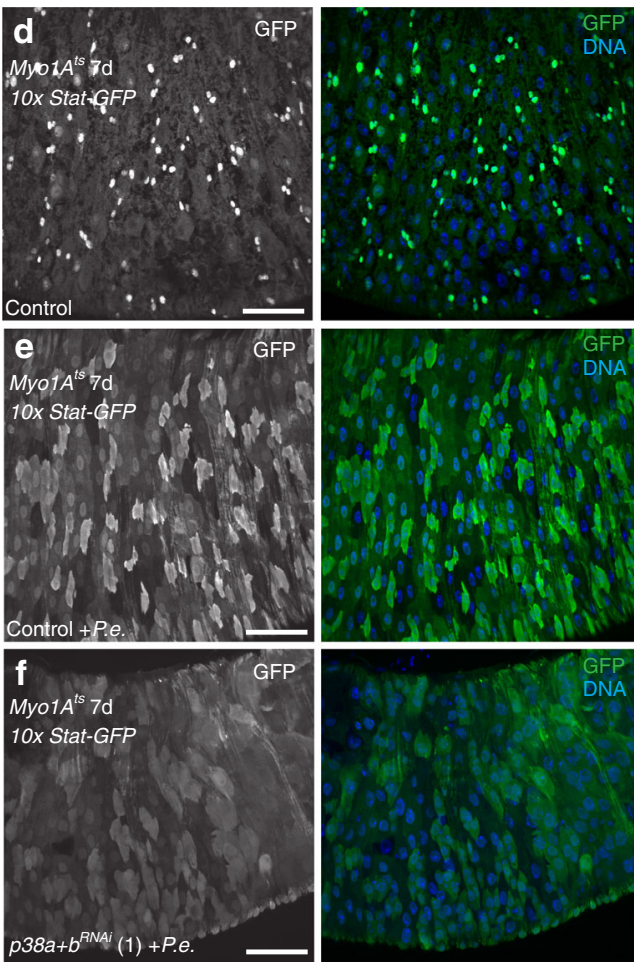

g Myo1A $A^{t s}, 10 \times$ STAT-GFP $7 \mathrm{~d}, 29^{\circ} \mathrm{C}$

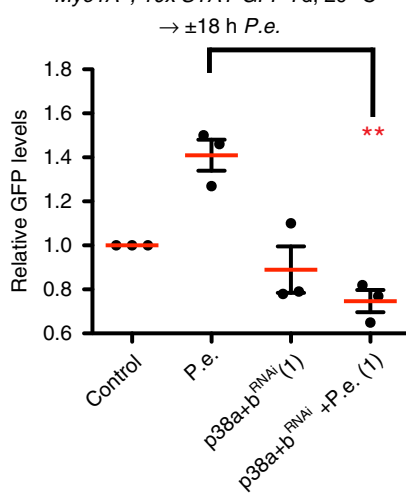

Fig. 2 Enterocyte p38 signaling promotes ISC proliferation via cytokine Upd3/Jak-Stat signaling. a Multiple stresses induce cytokine and JAK-STAT target gene expression. Mean relative mRNA with s.e.m. from $n=3$ independent experiments of cytokine upd3 mRNA levels (one-way ANOVA: $p<0.0001$; Dunnett's: P.e. $<0.0001, E c c 15=0.0323, \mathrm{H}_{2} \mathrm{O}_{2}=0.4896, \mathrm{SDS}=0.0089$, bleomycin $=0.409$, heat shock $=0.0392$ ) and JAK-STAT target, Socs36E, mRNA levels (one-way ANOVA: $p=0.0002$; Dunnett's: P.e. $<0.0001, E c c 15<0.0001, \mathrm{H}_{2} \mathrm{O}_{2}=0.3157$, SDS $<0.0001$, bleomycin $<0.0001$, heat shock $<$ 0.0001 ) determined by qPCR from stressed and unstressed $w^{1118}$ midguts. b p38 signaling promotes cytokine induction upon P.e. infection. Mean relative mRNA with s.e.m. from $n=3$ independent experiments of cytokine upd3 mRNA levels determined by qPCR from uninfected and infected midguts with p38b- (one-way ANOVA: $p=0.0223$; one sample $t$ test: $p=0.0452$ ) and $p 38 a+b$-depleted (one-way ANOVA: $p=0.0202$; one sample $t$ test: $p=$ $0.0421)$ in ECs for 7 days and control midguts $\left(M y 01 A^{t s}\right)$. c Overexpression of MKK3/Licorne in ECs promotes cytokine expression. Mean relative mRNA with s.e.m. from $n=3$ independent experiments of upd2 (one sample $t$ test: $p=0.0488$ ) and upd3 mRNA (one sample $t$ test: $p=0.0467$ ) levels determined by qPCR on midguts overexpressing Licorne for 1 day using EC-specific, Myo1 $A^{\text {ts }}$ and control midguts (Myo1 $\left.A^{t s}\right)$. d-g p38 signaling in enterocytes promotes Stat activity upon P.e. infection. Increased 10x STAT-GFP is observed in P.e.-infected control midguts (e right, green) compared with uninfected control midguts (d right, green). Reduced 10x STAT-GFP is observed however in P.e.-infected midguts expressing $p 38 a+b^{R N A i}$ in ECs for 7 days (f right, green) compared with P.e.-infected control midguts. Quantified GFP levels are in $\mathbf{g}(n=3$ midguts from one experiment; unpaired $t$ test; $p=$ 0.0017). DNA is in blue. Scale bars in $\mathbf{d}-\mathbf{f}$ are $50 \mu \mathrm{m}$. Representative images in $\mathbf{d}-\mathbf{g}$ from three experiments. Source data are provided as a Source Data File

Ask1 activates p38 upon stress and promotes regeneration. Both JNK and p38 are known to be activated upon stress via upstream SAP3Ks (i.e., Mekk1, Ask1, and Tak1) and SAP2Ks (i.e., MKK4/7 and MKK3/6) ${ }^{13}$. Previously it was reported that p38 was activated in fly ECs via Mekk1 and MKK3 after feeding flies soluble microbial extract ${ }^{21}$. Surprisingly, neither p38 activation nor the ISC response to infection was inhibited upon P.e. infection in Mekk1 mutant midguts (Supplementary Fig. 6a-e) or in midguts depleted for Mekk1 by RNAi in ECs with Myo1 ${ }^{\text {ts }}$ (Supplementary Fig. 6f-m). Since Tak1 signaling is important for 

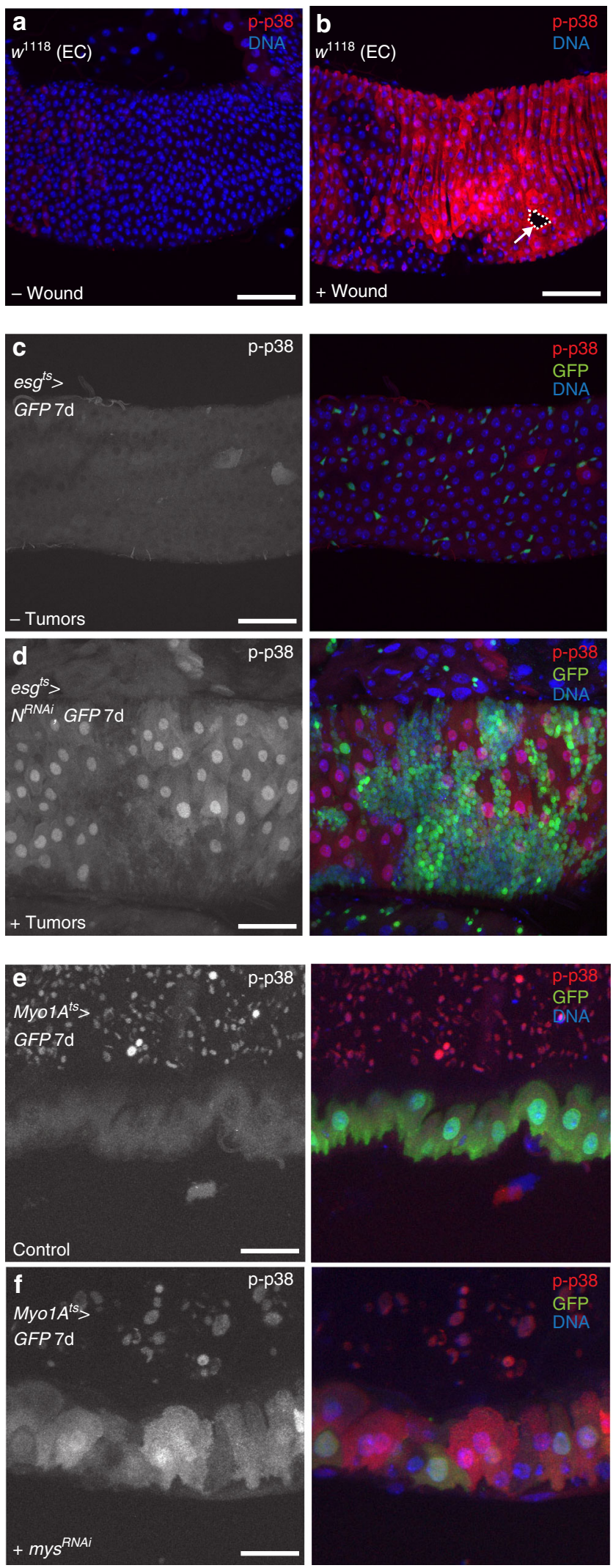

AMP production after pathogenic infection ${ }^{31}$, we tested if Tak1 was required for p38 activation in ECs upon infection. We found that neither p38 activation nor the ISC response upon infection are affected in Tak1 mutant $\left(T a k 1^{2}\right)$ midguts (Supplementary Fig. 7a-e). However, basal p38 levels in Tak1 mutant midguts
Fig. 3 Wounding, tumor growth, and cell detachment activate p38 signaling in ECs. a, b Wounding with a needle activates p38 signaling in enterocytes (ECs) (b red) compared with unwounded midgut ECs (a). Wound site is indicated with an arrow and outlined with dashed line. c, d Tumor growth activates p38 signaling in surrounding intestinal epithelial cells. p38 signaling is activated in ECs of midguts expressing $N^{R N A i}$ and GFP in progenitors for 7 days with esg ${ }^{\text {ts }}>$ GFP (d right, red), but not in tumor-free midguts expressing GFP alone in progenitors for 7 days with esg $g^{\text {ts }}>$ GFP (c right, red). e, $\mathbf{f}$ Intestinal epithelial cell detachment from the basement membrane activates p38 signaling. p38 signaling is increased in detaching ECs of midguts expressing mys ${ }^{R N A i}$ and GFP for 7 days with Myo1 $A^{\text {ts }}$ (f right, red) compared with midguts expressing GFP alone in ECs for 7 days with Myo1A ${ }^{\text {ts }}$ (e right, red). DNA is in blue. Scale bars in a-d are $50 \mu \mathrm{m}$; in e, $\mathbf{f}, 20 \mu \mathrm{m}$. Representative images in $\mathbf{a}$ and $\mathbf{b}$ from three experiments; c, d, e, f, two experiments

were detectably higher relative to unstressed controls (Supplementary Fig. 7a, c). Since ROS production via Duox has been proposed as a defense to bacterial infection, we tested whether Ask1, a SAP3K thought to sense ROS ${ }^{32}$, was required for p38 activation in ECs. Ask1 was previously shown in C. elegans to be required for resistance to pathogenic infection ${ }^{20}$; however, how Ask1 promotes resistance and its requirement in gut epithelial regeneration is unknown. Basal levels of active p38 were not measurably affected in unstressed Ask1 mutant (Ask1 ${ }^{\text {MB06487) }}$ midguts, or midguts depleted for Ask1 in ECs, or expressing an EC-targeted catalytically dead form of Ask1 (Ask1 $\left.{ }^{\mathrm{K} 618 \mathrm{M}}\right)^{42}$ (Figs. 4a, d, 5a, d, h, j; Supplementary Fig. 8a-d, f, h). However, we found that these treatments all strongly suppressed p38 activation during P.e. infection (Fig. 5b, c, e, f; Supplementary Fig. 8e, $\mathrm{g}, \mathrm{i})$. Ask1 was also required for p38 activation in ECs after $1 \%$ $\mathrm{H}_{2} \mathrm{O}_{2}$ or $0.2 \%$ SDS exposure relative to stressed control midguts (Fig. 4b, c, e, f). Next, we determined whether Ask1 was also required in ECs for the ISC response to damage. While Ask1 depletion alone did not detectably affect basal ISC divisions, Ask1 depletion in ECs impaired the ISC proliferation response to P.e. infection (Fig. 5g). Furthermore, we found that Ask1 depletion in ECs suppressed p38 activation in ECs, but not the VM, upon wounding (Fig. 5h-k; Supplementary Fig. 9c-j). These data indicate that Ask1 is required for p38 activation upon chemical stress, mechanical stress, and pathogenic bacterial infection. Furthermore, they suggest that Ask1-p38 signaling but not Mekk1- or Tak1-p38 signaling is required in ECs for the ISC response to damage.

Nox-derived ROS activate $\mathrm{p} 38$ and promote midgut regeneration. Since ingested ROS could activate Ask1-p38 in midgut ECs, we next tested whether ROS were produced in response to different stresses. Using qPCR, we found that the Nrf2 target, GstD1, was induced upon a variety of stresses, suggesting an increase in ROS levels in stressed midguts relative to unstressed midguts (Fig. 6f). Since gut-produced ROS contributes to innate immunity and ISC proliferation in the fly midgut ${ }^{10,26}$, we next tested whether ROS were required for p38 activation after pathogenic infection. To decrease ROS in ECs, we overexpressed Nrf2 (Cnc), a transcription factor that promotes antioxidant factor expres$\operatorname{sion}^{43}$. As an alternative, we co-overexpressed the antioxidant enzymes superoxide dismutase 1 ( $\mathrm{Sod} 1$ ), which converts superoxide $\left(\mathrm{O}_{2} \bullet\right)$ to $\mathrm{H}_{2} \mathrm{O}_{2}$, and Catalase, which converts $\mathrm{H}_{2} \mathrm{O}_{2}$ to $\mathrm{H}_{2} \mathrm{O}$ and $\mathrm{O}_{2}$. While overexpression of these factors alone moderately increased active p38 levels and ISC proliferation without infection (Fig. 6a, d, e; Supplementary Fig. 10a, b, d), overexpression of these factors inhibited p38 activation during P.e. infection (Fig. 6b, c; Supplementary Fig. 10c). Furthermore, the ISC response to P.e. infection and detergent exposure was strongly 

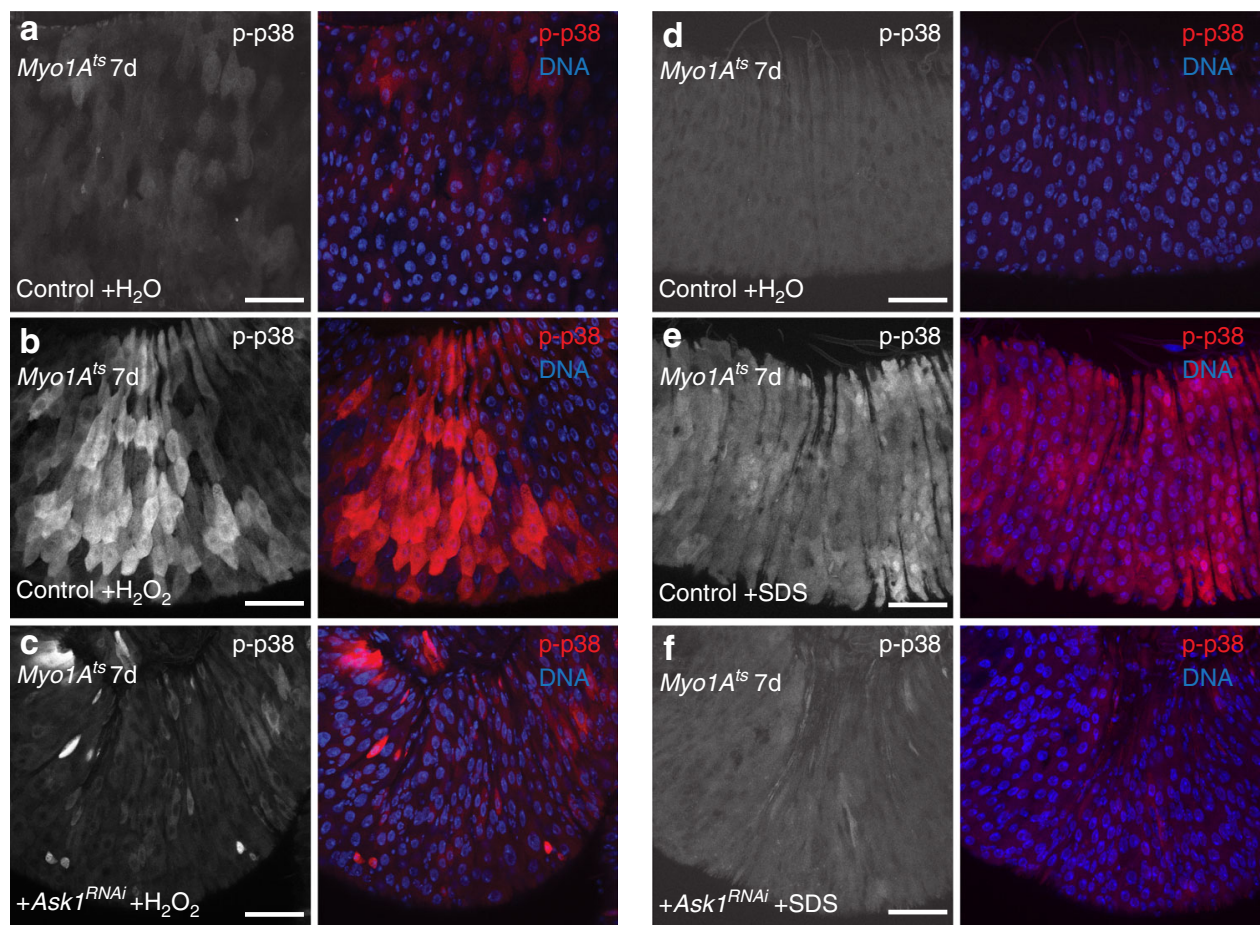

Fig. 4 Ask1-p38 signaling is activated in ECs after ROS and detergent damage. a-f Ask1 is required for p38 activation in $E C s$ upon $\mathrm{H}_{2} \mathrm{O}_{2}$ and detergent exposure. p38 activation increases in ECs in $\mathrm{H}_{2} \mathrm{O}_{2}$ or SDS-fed control (EC-specific, Myo1A ${ }^{\text {ts}}$ ) midguts (b, e right, red) compared with $\mathrm{H}_{2} \mathrm{O}$-fed control midguts (a, d right, red). p38 activation is blocked, however, in $\mathrm{H}_{2} \mathrm{O}_{2}$ or SDS-fed midguts expressing Ask $7^{R N A i}$ in ECs for 7 days with Myo1A ${ }^{\text {ts }}$ (c, $\mathbf{f}$ right, red) compared with $\mathrm{H}_{2} \mathrm{O}_{2}$ or SDS-fed control midguts. DNA is in blue. Scale bars in a-f are $50 \mu \mathrm{m}$. Representative images from three experiments

suppressed relative to control by Nrf2 overexpression (Fig. 6d, e), suggesting that ROS are required in ECs to promote ISCmediated regeneration in response to a variety of damage and not only to pathogens. To identify the molecular source of ROS production, we depleted Nox or Duox, which produce $\mathrm{O}_{2} \bullet$ and $\mathrm{H}_{2} \mathrm{O}_{2}$, respectively, and contribute to mucosal immunity through ROS generation $22,26,44$. Depletion of Nox or Duox in ECs of unchallenged midguts neither detectably affected basal active p38 levels nor the basal rate of ISC division (Fig. 7a, d, e; Supplementary Fig. 11a, b, d, f). However, Nox depletion was able to inhibit p38 activation during P.e. infection relative to infected controls (Fig. 7b, c). Duox depletion in ECs did not detectably affect p38 activation upon P.e. infection (Supplementary Fig. 11c, e). These data suggest that p38 is activated by Nox-derived ROS produced during pathogenic infection. We then determined if ROS generated during pathogenic infection are required in ECs for ISC proliferation. We found that depletion of either Nox or Duox in ECs partially suppressed the normal proliferative response in ISCs relative to infected control midguts (Fig. 7d; Supplementary Fig. 11f). These data suggest that in addition to promoting pathogen clearance, ROS produced by both Nox and Duox in ECs promote intestinal regeneration. In addition, we found that Nox was required in ECs for optimal ISC responses following detergent damage and p38 activation upon wounding (Fig. 7e-h; Supplementary Figs. 11g, 12). Nox-derived ROS appears to promote regeneration in response to a variety of stresses. Interestingly, we found that Duox was not required in ECs for intestinal regeneration following detergent damage (Supplementary Fig. 11g), suggesting that Duox activity may be specific for pathogen clearance. Together these data suggest that Nox can sense diverse types of damage and stress in ECs, and that Nox-derived ROS promote intestinal regeneration by activating Ask1-MKK3-p38 signaling.

\section{Discussion}

A role for p38 has been reported in intestinal immunity ${ }^{1}$ and in mouse intestinal regeneration upon detergent-induced damage 24,25 . However, how p38 senses stress and promotes intestinal regeneration is not well understood. We found that p38 signaling is activated in ECs during pathogenic bacterial (P.e.) infection, oxidative stress $\left(\mathrm{H}_{2} \mathrm{O}_{2}\right)$, detergent exposure (SDS), wounding, tumor growth, and EC detachment from the BM. In addition, we found that the SAP2K MKK3/Licorne and the SAP3K, Ask1, were required to activate p38 in ECs during P.e. infection. Moreover, Ask1 was required for p38 activation in ECs after oxidative stress $\left(\mathrm{H}_{2} \mathrm{O}_{2}\right)$, detergent (SDS) exposure, and wounding, suggesting that Ask1 may act as a node that integrates multiple types of stress inputs for p38 activation. Using Nrf2 and antioxidant (Sod1 and Catalase) overexpression, we also found that ROS are required for p38 activation during P.e. infection. Furthermore, we determined that ROS contributed by the NADPH oxidase, Nox, were required for full p38 activation upon P.e. infection and wounding. Surprisingly, Duox, previously also suggested to produce ROS to promote intestinal immunity and repair ${ }^{10,22}$, was not detectably required for p38 activation. Furthermore, Nox, Ask1, and p38 were all required in ECs to stimulate ISC proliferation and regeneration following P.e. infection. This stem cell response appeared to be mediated at least in part by Upd3/Jak-Stat signaling. Lastly, Nox-derived ROS was also required for ISCmediated regeneration upon detergent exposure and p38 activation upon wounding, suggesting that Nox, like Ask1, may also sense multiple different types of epithelial stresses to promote regeneration. Our work suggests that in addition to JNK and Hpo signaling, Nox-Ask1-MKK3-p38 signaling promotes intestinal regeneration in response to multiple different types of stress, thereby enhancing tissue and organismal 

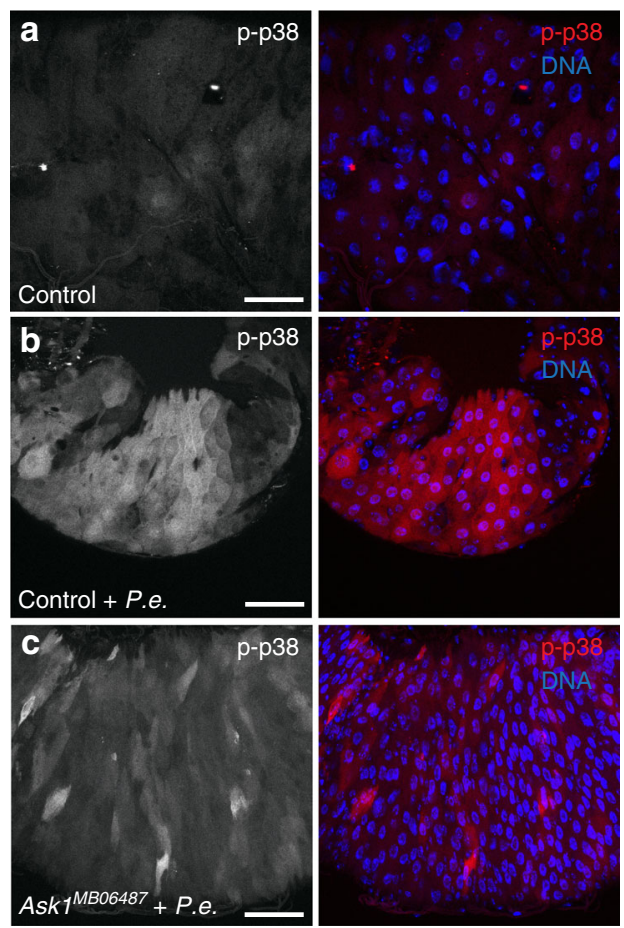

g

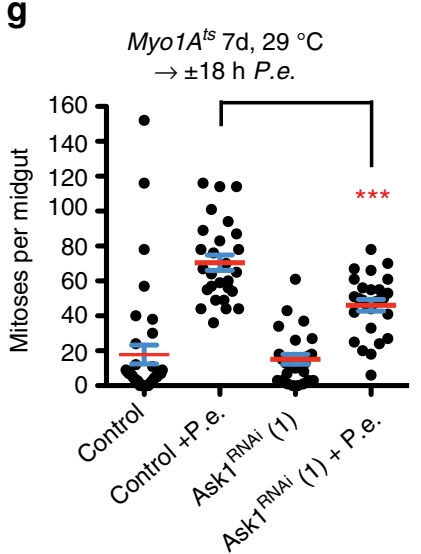

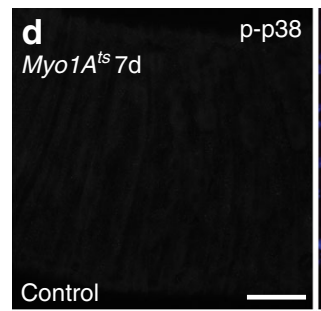
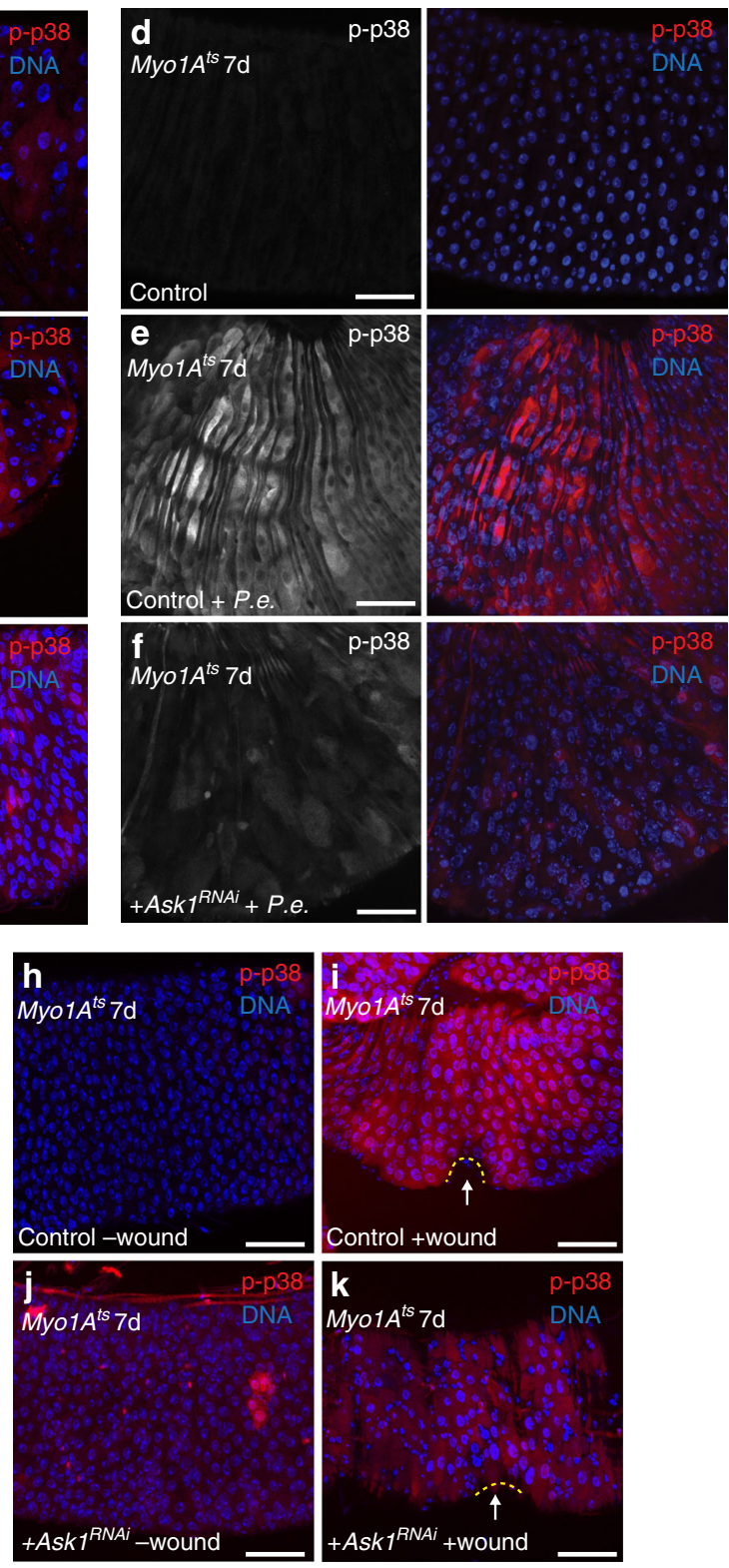

Fig. 5 Ask1-p38 signaling in ECs promotes ISC proliferation upon damage. a-c Ask1 is required for p38 activation in ECs upon P.e. infection. p38 activation is increased in ECs in P.e.-infected control midguts ( $\mathbf{b}$ right, red) compared with uninfected control midguts (a; right, red). p38 activation is blocked in infected Ask $7^{M B 06487}$ midguts (c right, red) compared with infected control midguts. d-f Ask1 is required for p38 activation in ECs upon P.e. infection. p38 activation is increased in ECs in P.e.-infected control (EC-specific, Myo1 $A^{t s}$ ) midguts (e right, red) compared with uninfected control midguts (d right, red). p38 activation is blocked, however, in P.e.-infected midguts expressing Ask $7^{R N A i}$ in ECs for 7 days with Myo1 $A^{\text {ts }}$ (f right, red) compared with P.e.-infected control midguts. $\mathbf{g}$ Ask1 is required in ECs to promote ISC proliferation upon P.e. infection. The mean number of phosphorylated histone H3 Ser 10-positive cells per midgut with s.e.m. in control midguts $\left(M y 01 A^{t s}\right)\left(n=37\right.$ midguts), P.e.-infected control midguts $\left(n=27\right.$ midguts), midguts expressing Ask $7^{R N A i}$ in ECs for 7 days with Myo1Ats ( $n=27$ midguts) and in P.e.infected midguts expressing $A s k 7^{R N A i}$ in ECs for 7 days ( $n=27$ midguts) (Mann-Whitney; $p=$ 0.0003). Midguts pooled from three independent experiments. h-k Ask1 is required for p38 activation in ECs upon wounding. p38 is activated in ECs of wounded control midguts (i red) compared with unwounded control midguts (h red) or unwounded midguts expressing Ask $7^{R N A i}$ in ECs for 7 days with Myo1 $A^{\text {ts }}$ ( $\mathbf{j}$ red). p38 activation is blocked in ECs of wounded midguts expressing Ask $7 N A A$ in ECs for 7 days with Myo1Ats ( $\mathbf{k}$ red) relative to ECs in wounded control midguts. DNA is in blue. Scale bars in a-f, h-k are $50 \mu \mathrm{m}$. Representative images in a-c from two experiments; $\mathbf{d}-\mathbf{f}$, three experiments; $\mathbf{h}-\mathbf{k}$, two experiments. Source data are provided as a Source Data File

resilience (Fig. 8). Moreover, since NADPH oxidases (Nox, Duox) are expressed in a variety of cell types, including macrophages ${ }^{45}$, and since Ask1-p38 signaling can potentially be activated in any cell type, our work suggests that Nox-Ask1MKK3-p38 signaling may promote regeneration in a variety of contexts.

Previous work in the fly midgut reported that Mekk1-p38aAtf2 and Mekk1-p38c-Atf2 activity promote intestinal immunity by inducing Duox-mediated ROS production ${ }^{22,23}$, and that ROS are required for intestinal regeneration ${ }^{10}$. However, we found that Mekk1, p38a, p38c, and Atf2 are not required for intestinal regeneration. Instead, we did find that both $p 38 a+b$ are required for intestinal regeneration, partly by regulating upd3 expression. Interestingly, Duox was also required upon P.e. infection for robust intestinal regeneration, suggesting that additional mechanisms may increase Duox activity and that its increased 

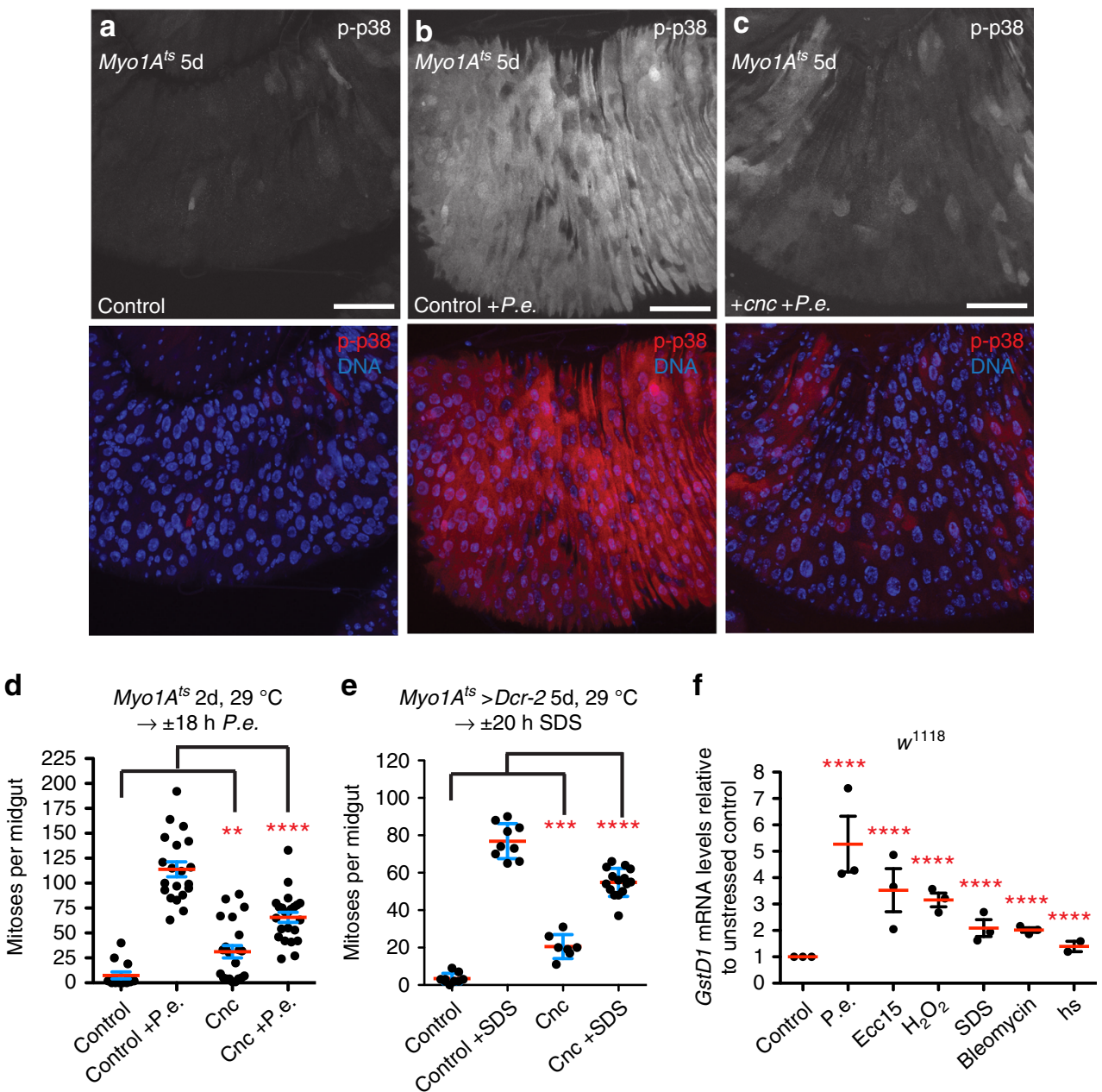

Fig. 6 ROS-p38 signaling in ECs promotes ISC proliferation upon damage. a-c ROS are required for p38 activation in ECs upon P.e. infection. p38 activation increases in ECs in P.e.-infected control midguts (EC-specific, Myo1Ats) (b below, red) compared with uninfected control midguts (a below, red). p38 activation is blocked, however, in P.e.-infected midguts overexpressing Nrf2 (Cnc) in ECs for 5 days with Myo1A ${ }^{\text {ts }}$ (c below, red) compared with P.e.infected control midguts. d, e Nrf2 overexpression in ECs blocks ISC-mediated regeneration upon P.e. infection and detergent exposure. The ISC response is blocked in P.e.-infected midguts overexpressing Nrf2 in ECs for 2 days with Myo1 ${ }^{t s}$ compared with P.e.-infected control midguts (Mann-Whitney; $p<$ 0.0001). ISC mitoses are increased in midguts overexpressing Nrf2 for 2 days compared with control midguts (Mann-Whitney; $p=0.0011$ ). The mean number of phosphorylated histone $\mathrm{H} 3$ Ser 10-positive cells per midgut with s.e.m. in control midguts (Myo1A $\left.A^{\text {ts }}\right)(n=13$ midguts), P.e.-infected control midguts ( $n=20$ midguts), midguts expressing Nrf2 (cnc) in ECs for 2 days with Myo1A $A^{\text {ts }}(n=22$ midguts) and in P.e.-infected midguts expressing cnc in ECs for 2 days ( $n=23$ midguts). Midguts pooled from two independent experiments. d ISC-mediated regeneration is blocked in SDS-fed (20 h) midguts overexpressing Nrf2 ( $\mathrm{Cnc})$ in ECs for 6 days with Myo1 $A^{\text {ts }}$ compared with SDS-fed control midguts (unpaired $t ; p<0.0001$ ). ISC mitoses are increased in midguts overexpressing Nrf2 for 6 days compared with control midguts (unpaired $t ; p 0.0001$ ). The mean number of phosphorylated histone H3 Ser 10-positive cells per midgut with s.e.m. from one representative experiment in control midguts (Myo1Ats $>$ Dcr-2) $(n=9$ midguts), SDS-fed control midguts ( $n=9$ midguts), midguts expressing Nrf2 ( $C n c)$ in ECs for 6 days with Myo1Ats ( $n=7$ midguts) and in SDS-fed midguts expressing Cnc in ECs for 6 days ( $n=15$ midguts) (e). f Multiple stresses induce ROS-Nrf2 target gene GstD1 expression. Mean relative GstD1 mRNA levels with s.e.m. from $n=3$ independent experiments (one-way ANOVA: $p=0.0086$; Dunnett's: P.e. $<0.0001$, Ecc $15<0.0001, \mathrm{H}_{2} \mathrm{O}_{2}<0.0001$, SDS $<0.0001$, bleomycin $<0.0001$, heat shock $<0.0001$ ) determined by qPCR from stressed and unstressed $w^{1118}$ midguts. DNA is in blue. Scale bars in a-c are $50 \mu \mathrm{m}$. Representative images in a-c from three experiments. Source data are provided as a Source Data File

activity upon infection is not solely through its transcriptional upregulation via Atf2. Previous work showed that the survival of infected $p 38 a$ or $p 38 b$ mutant flies upon P.e. infection was comparable with control infected flies ${ }^{23}$. We similarly found that the ISC response to infection in $p 38 a$ and $p 38 c$ double-mutant $\left(p 38 a^{1}\right)$ midguts or those depleted for $p 38 a$ in ECs were similar to control infected midguts. These data suggest that neither $\mathrm{p} 38 \mathrm{a}$ nor $\mathrm{p} 38 \mathrm{c}$ alone are required for intestinal regeneration. Instead, the combination of $p 38 a$ and $p 38 b$ is required in ECs to promote ISC-mediated regeneration. While $p 38 b$ mutant midguts did show a mild defect in the ISC response to infection, loss of $p 38 b$ alone may not reduce organismal resilience to infection. Previous work suggests that p38b partially regulates upd3 expression ${ }^{19}$. Consistent with this, we found that $u p d 3$ induction is blocked upon infection in midguts expressing $p 38 b^{R N A i}$ or $p 38 a+b^{\mathrm{RNAi}}$ in ECs, and that JAK-STAT signaling is blocked in midguts expressing $p 38 a+b^{R N A i}$ in ECs. Together these data suggest that $\mathrm{p} 38 \mathrm{a}+\mathrm{b}$ signaling promotes intestinal regeneration upon infection via Upd3/JAK-STAT signaling, and not through Atf2mediated Duox expression.

Like p38, JNK in ECs has been shown to sense stress, i.e., pathogenic bacteria, ROS, tumor growth, and EC detachment 

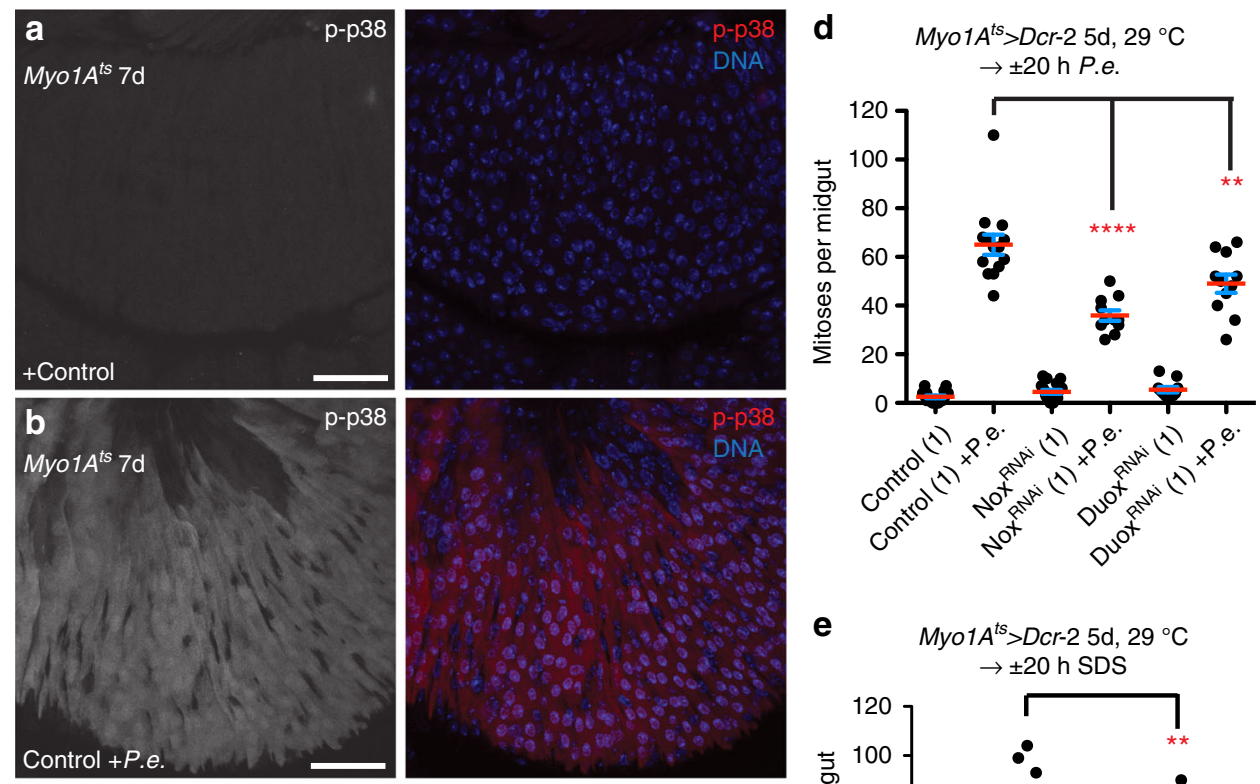
e $\quad$ Myo $1 A^{t s}>D c r-25 \mathrm{~d}, 29^{\circ} \mathrm{C}$ $\rightarrow \pm 20 \mathrm{~h}$ SDS
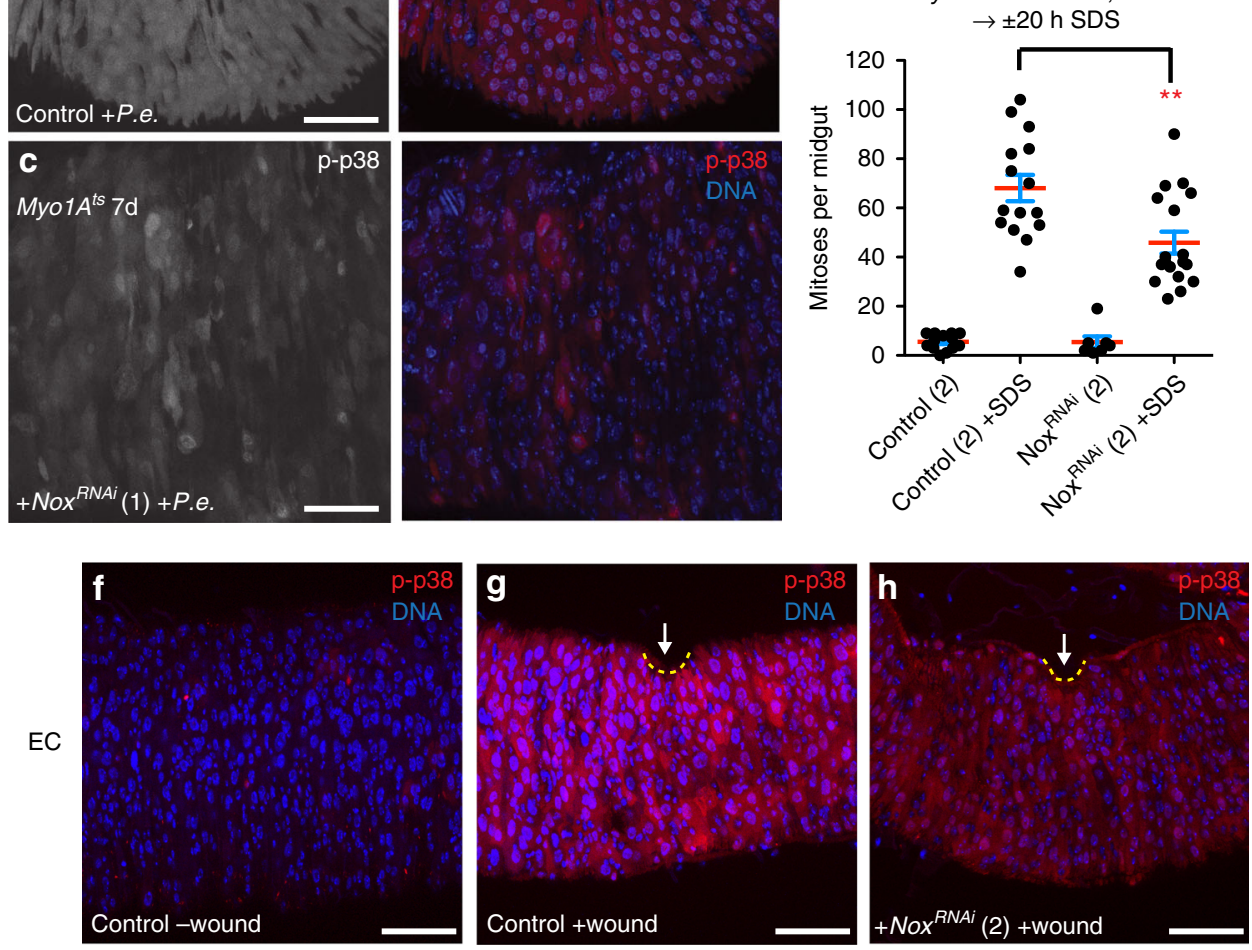

Fig. 7 Nox-derived ROS promotes p38 activation and intestinal regeneration. a-c Nox is required for p38 activation in ECs upon P.e. infection. p38 activation increased in ECs of P.e.-infected control (Myo1 $A^{t s}$ ) midguts ( $\mathbf{b}$ right, red) compared with uninfected control midguts (a right, red). p38 activation is blocked, however, in P.e.-infected midguts expressing Nox ${ }^{R N A i}$ in ECs for 7 days with Myo1A ${ }^{\text {ts }}$ (c right, red) compared with P.e.-infected control midguts. d Nox and Duox are both required for ISC-mediated regeneration upon P.e. infection. The ISC response is blocked in P.e.-infected midguts expressing Nox ${ }^{R N A i}$ (1) or $D_{u o x}^{R N A i}$ (1) for 5 days with Myo1Ats $>$ Dcr-2 compared with P.e.-infected control midguts (unpaired $t ; p<0.0001$ (Nox $\left.{ }^{R N A i}\right), p<0.0103$ (Duox $\left.{ }^{R N A i}\right)$. The mean number of phosphorylated histone H3 Ser 10-positive cells per midgut with s.e.m. from one representative experiment in control (1) midguts $(n=18$ midguts) (Myo1Ats $>$ Dcr-2 alone), P.e.-infected control (1) midguts ( $n=14$ midguts), midguts expressing Nox ${ }^{R N A i}(1)$ in ECs for 5 days with Myo1 ${ }^{\text {ts }}>$ Dcr-2 $\left(n=19\right.$ midguts), P.e.infected midguts expressing Nox ${ }^{R N A i}(1)$ in ECs for 5 days ( $n=11$ midguts), midguts expressing Duox ${ }^{R N A i}$ ( 1 ) in ECs for 5 days with Myo1 $A^{\text {ts }}>\operatorname{Dcr}-2$ ( $n=11$ midguts) and P.e.-infected midguts expressing Duox RNAi ( 1 ) in ECs for 5 days with MyolAts $>$ Dcr-2 ( $n=11$ midguts). e Nox is required in ECs for ISC-mediated regeneration upon detergent exposure. The ISC response is blocked in SDS-fed (20 h) midguts expressing Nox ${ }^{R N A i}$ (2) for 5 days with Myo1Ats $>$ Dcr-2 compared with SDS-fed control midguts (unpaired $t$ test; $p<0.0001$ ). The mean number of phosphorylated histone H3 Ser 10 positive cells per midgut with s.e.m. in control (2) midguts ( $n=17$ midguts from one representative experiment) (Myo1Ats $>D c r-2$ alone), SDS-fed control (2) midguts ( $n=29$ midguts from two experiments), midguts expressing Nox ${ }^{R N A i}$ (2) in ECs for 5 days with Myo1A ${ }^{\text {ts }}>$ Dcr-2 ( $n=6$ midguts from one representative experiment), and in SDS-fed midguts expressing Nox ${ }^{R N A i}(2)$ in ECs for 5 days ( $n=32$ midguts from two experiments). f-h Nox-derived ROS are required for p38 activation in ECs upon wounding. p38 is activated in ECs of wounded control midguts ( $\mathbf{g}$ red) compared with unwounded control midguts ( $\mathbf{f}$ red). p38 activation is blocked in ECs of wounded midguts expressing Nox ${ }^{R N A i}$ (2) (h red) relative to ECs in wounded control midguts. DNA is in blue. Scale bars in a-c, $\mathbf{f}-\mathbf{h}$ are $50 \mu \mathrm{m}$. Representative images in $\mathbf{a}-\mathbf{c}$ from three experiments; $\mathbf{f}-\mathbf{h}$, two experiments. Source data are provided as a Source Data File 


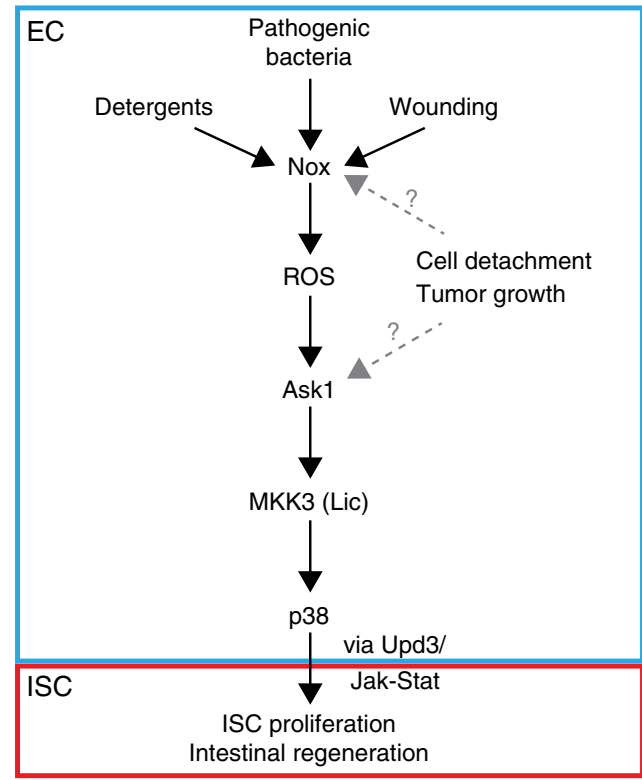

Fig. 8 Damage sensing by Nox-Ask1-MKK3-p38 signaling in the fly intestinal epithelial niche. Multiple stresses (pathogenic bacteria, detergents, and wounding) stimulate reactive oxygen species (ROS) production by NADPH oxidase (Nox), thus activating Ask1-MKK3-p38 signaling in enterocytes (EC). Cell detachment and tumor growth may also activate MKK3-p38 signaling either via Nox or Ask1. p38 signaling stimulates Upd3 expression in ECs and JAK-STAT activity in intestinal stem cells (ISCs), thus boosting ISC proliferation and supporting intestinal regeneration

from the BM. However, unlike p38, JNK can sense heat stress, and heat stress can induce $u p d 3$ expression. While JNK activation in ECs had previously been shown to be sufficient to promote cytokine upd2-3 expression and ISC proliferation ${ }^{8}$, whether JNK is required in ECs for intestinal regeneration had, surprisingly, not been directly demonstrated. We found that JNK signaling is indeed required in ECs for normal ISC-mediated regeneration upon P.e. infection (Supplementary Fig. 13a). In addition to JNK, Hpo signaling is inhibited in response to a variety of stresses and required for intestinal regeneration ${ }^{7,11,12}$. Since depleting Nox, Ask1, or p38 in ECs only partially suppressed regeneration, it seems likely that Nox-Ask1-p38 signaling works together with JNK and/or Hpo/Yki signaling to promote mitogen production and ISC activation. Alternatively, p38 might act via Yki, activating it in ECs by antagonizing Hpo/Wts signaling ${ }^{46}$. To test whether p38 and JNK work together, we depleted $p 38 a+b$ with $R N A i$ and overexpressed the JNK inhibitor, puckered in ECs. We found that blocking $\mathrm{p} 38 \mathrm{a}+\mathrm{b}$ and JNK with these treatments did not inhibit the ISC response to P.e. infection relative to infected controls. Furthermore, we found increased basal ISC divisions without infection in midguts with ECs with reduced p38a $+\mathrm{b}$ and JNK activity, confounding our interpretation of this experiment. The details of how these stress-sensing pathways (Jnk, p38, and Hpo) cross-talk and integrate stress responses in the midgut remain an interesting area for future studies.

We found that many different types of stress can activate p38 signaling in adult fly midgut ECs, i.e., Gram-negative pathogenic bacteria (P.e.), ROS $\left(\mathrm{H}_{2} \mathrm{O}_{2}\right)$, detergent (SDS), wounding, ISC tumor growth and $\mathrm{EC}$ detachment. Furthermore, we found that Gram-negative pathogenic bacteria, ROS, detergents, and wounding all require Ask1 to activate p38 in ECs. These data suggest that a variety of stresses can be sensed in ECs by Ask1. How Ask1 senses these stresses is not well understood. Ask1 could possibly sense each stress by distinct mechanisms. On the other hand, each stress might also trigger ROS production, possibly via Nox, and thereby activate Ask1. Consistent with this mechanism, we found that a ROS-responsive Nrf2 target, GstD1, is induced after infection, detergent, oxidative, DNA damage, and heat shock stress, suggesting that ROS are produced by all these stresses. Furthermore, Nox-derived ROS were required in ECs for p38 activation upon infection and wounding, and for the ISC response to infection and detergent exposure. Thus our data are consistent with the possibility that Ask1 senses damage through Nox-derived ROS to effect intestinal regeneration. Ask1 proteins have been proposed to oligomerize using their C-terminal coiled-coiled domains ${ }^{47}$. Ask1 activity is thought to be regulated by ROS at its $\mathrm{N}$-terminal coil-coil domain, which binds to the redox protein thioredoxin ${ }^{32}$. Thioredoxin binding to the $\mathrm{N}$ terminal coil-coil domain of Ask1 is thought to prevent autophosphorylation and activation of oligomerized Ask $1^{47}$. Upon oxidative stress, ROS oxidize thioredoxin, causing thioredoxin to dissociate from Ask1 and resulting in the activation of oligomerized Ask1 proteins ${ }^{47}$. For this reason, an $\mathrm{N}$-terminally deleted Ask1 $\left(\triangle \mathrm{N}\right.$ Ask1) is thought to be constitutively active ${ }^{32}$. An alternate model proposes that Ask1 oxidation results in oligomerization via intermolecular cysteine disulfide bond formation and activation through phosphorylation ${ }^{48}$. In addition, oligomerization has been proposed to be dependent on multiple cysteines throughout Ask1, and interestingly, $\Delta \mathrm{N}$ Ask1 was able to oligomerize and activate $\mathrm{JNK}^{48}$. Furthermore, disulfide bond formation between thioredoxin and Ask1 is thought to result in rapid reduction of Ask1 oligomers to an active monomeric form ${ }^{48}$. However, we found that overexpression of $\Delta \mathrm{N} \mathrm{Ask} 1^{49}$ in ECs did not act as a constitutively active form of Ask1 and was unable to strongly activate p38, raising questions about these proposed mechanisms. Surprisingly, we were able to suppress p38 activation during P.e. infection by expressing the $\Delta \mathrm{N}$ Ask1. While we show that Ask1 plays an important role in stress-triggered intestinal regeneration, precisely how these stresses activate Ask1 remains to be determined.

ROS produced by the NADPH oxidase, Duox, in ECs have been reported to promote not only intestinal immunity toward pathogenic bacteria but also intestinal regeneration ${ }^{10,22}$. Similarly, another NADPH oxidase, Nox, also in ECs, has been reported to produce ROS upon exposure to specific commensal bacteria, which subsequently also stimulates ISC proliferation ${ }^{26}$. Nevertheless, whether Nox-derived ROS provides immunity toward either commensal or pathogenic bacteria or whether it normally promotes intestinal turnover remained to be determined. We found that Nox was required in ECs even more than Duox to activate p38 and promote ISC proliferation in response to pathogenic bacteria. This is consistent with our finding that Nox, but not Duox, mRNA is strongly upregulated $(18 \times)$ in ECs after P.e. infection ${ }^{50}$. Our data suggest that Nox plays an important role, possibly as important as Duox, in intestinal immunity and regeneration during pathogenic bacterial infection. It is thought that ROS can directly activate ISC proliferation upon stress ${ }^{51}$. However, our work suggests that a significant contributor to ISCmediated regeneration is nonautonomous activation of JAKSTAT signaling in ISCs by Nox-ROS-p38 signaling in $\mathrm{ECs}^{52}$. Furthermore, our data suggest that unlike Duox, Nox plays an important role in intestinal regeneration following diverse types of damage. An important question remaining is how ROS production by Nox is activated by each stress. Nox could be stimulated by a single cue, such as actin ${ }^{53}$ or by different cues activated by each stress.

Inflammatory bowel diseases (e.g., ulcerative colitis, Crohn's disease) are characterized by chronic gastrointestinal inflammation due to damage, and have been linked to an increased risk for colorectal cancer. The cause of IBDs and how they develop is 
unfortunately not well understood. Work in the mouse intestine indicates that p38a signaling is required for intestinal repair after detergent (DSS)-induced damage ${ }^{24,25}$. Furthermore, loss of p38a in the intestinal epithelium enhances carcinogen/DSS damageinduced intestinal tumor initiation ${ }^{24,54}$. Together with our work, these data indicate the importance of the damage sensing and pro-regenerative roles of p38 signaling in intestinal epithelial homeostasis. Thus, a better understanding of how damage is sensed by epithelia and coupled to tissue repair will hopefully yield insights important for the treatment of inflammatory diseases and cancer prevention.

Methods
Fly stocks. All experiments were performed using 5-10 -day old, adult, mated female Drosophila melanogaster unless stated otherwise. The following fly stocks were used: $w^{1118}, y w$, puc-lacZ, upd3-lacZ, 10x STAT-GFP, esgGAL4; tubGAL80 ${ }^{\text {ts }}$ UAS-GFP (esg $\left.g^{t s}\right), e s g G A L 4$ tubGAL80 ts UAS-GFP; UAS-FLP, act $<C D 2>$ GAL4

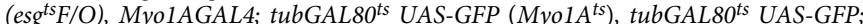
how (24B) GAL4 (how ${ }^{\text {ts }}$ ), $p 38 a^{1} 23$ and $p 38 b^{e x 9}$; UAS-bsk ${ }^{R N A i 55}$ (34138 GD), UASDuox ${ }^{R N A i}(2)(2593 \mathrm{GD})^{23,26}$, UAS-hep ${ }^{R N A i}$ (47507 GD), UAS-lic ${ }^{\text {RNAi }}$ (2)(106822 KK), UAS-Mekk1 ${ }^{R N A i}$ (2)(110339 KK), UAS-mys ${ }^{R N A i}(29619 \text { GD })^{9,56}$, UAS$\operatorname{Notch}^{R N A i}(27228 \mathrm{GD})^{9}$, UAS-Nox $^{R N A i}(2)(4914 \mathrm{GD})^{26}$, UAS-p38a $+b^{R N A i}((1)$ 34238 GD, (2) 52277 GD), UAS-p38b ${ }^{R N A i}$ (1)(108099 KK) from the Vienna Drosophila RNAi Center; UAS-Ask1 $1^{R N A i}$ (HMS00464), UAS-Atf2 ${ }^{R N A i}$ (HMC05118, JF02108), UAS-Dcr2, UAS-Duox ${ }^{R N A i}$ (1)(HMS00692), UAS-lic ${ }^{R N A i}(1)$ (HMS05002), UAS-Mekk1 ${ }^{R N A i}$ (1)(HM05075), UAS-Nox ${ }^{R N A i}$ (1)(HMS00691), UAS-p38aRNAi (1)(HMS01224), Ask1 $1^{M B 0648733}$ and Tak $1^{2} 31$ from the Bloomington Stock Center; UAS-p $38 b^{\text {antisense } 57}$ and UAS-p $38 b^{D N 57}$ from the Kyoto Stock Center; UAS-Ask $1^{K 618 M 42}$ from Masayuki Miura (University of Tokyo, Japan), Atf2 ${ }^{P B 34,41}$ from Shunsuke Ishii (RIKEN Tsukuba Institute), UAS-Cnc ${ }^{58}$ from Dirk Bohmann (University of Rochester, USA), UAS-lic from the Zürich ORFeome project (FlyORF), $M K 2^{\Delta l A}$ and $M K 2^{\Delta 43} 40$ from Hugo Stocker (ETH Zürich, Switzerland), Mekk1 ${ }^{U r 3621,23}$ from Shunsuke Ishii (RIKEN Tsukuba Institute), UAS-Sod1, UASCatalase $^{34}$ from Florenci Serras (University of Barcelona, Spain).

Stress experiments. P.e. infection: flies were treated for $18 \mathrm{~h}$ with food laced with either $500-650 \mu \mathrm{l}$ of $5 \%$ sucrose or $20 \times$ P.e. resuspended in $5 \%$ sucrose from an $18 \mathrm{~h}$ culture grown at $30^{\circ} \mathrm{C}, 130 \mathrm{RPM}$ or treated on filter paper with either $450 \mu \mathrm{l}$ of $5 \%$ sucrose or $5 \%$ sucrose containing P.e. resuspended from a $20-24 \mathrm{~h}$ culture grown at $29^{\circ} \mathrm{C}, 200 \mathrm{RPM}$. Erwinia caratova caratova 15 (Ecc15) infection: flies were treated on the filter paper with either $450 \mu$ of $5 \%$ sucrose or $5 \%$ sucrose containing $E c c 15(\mathrm{OD}=200)$ resuspended from a $20-24 \mathrm{~h}$ culture grown at $29^{\circ} \mathrm{C}$, 200 RPM. Hydrogen peroxide exposure: $1 \%$ final of stabilized $\mathrm{H}_{2} \mathrm{O}_{2}$ (SigmaAldrich) or $\mathrm{H}_{2} \mathrm{O}$ was mixed into food or fed on filter paper soaked with $450 \mu \mathrm{l}$ of either $5 \%$ sucrose or $0.8 \% \mathrm{H}_{2} \mathrm{O}_{2}$ in $5 \%$ sucrose. Detergent exposure: $0.2 \%$ sodium dodecyl sulfate (SDS) or $\mathrm{H}_{2} \mathrm{O}$ was mixed into food or fed on the filter paper soaked with $450 \mu \mathrm{l}$ of either $5 \%$ sucrose or $0.5 \%$ SDS in $5 \%$ sucrose. DNA damage: flies were fed on filter paper soaked with $450 \mu \mathrm{l}$ of either $5 \%$ sucrose or $500 \mu \mathrm{M}$ bleomycin sulfate $(\mathrm{Abcam})$ in $5 \%$ sucrose. Heat shock: flies were heat shocked for $30 \min$ or $4 \mathrm{~h}$ at $37^{\circ} \mathrm{C}$.

Drosophila genetics. Flies raised at $18^{\circ} \mathrm{C}$ were shifted to $29^{\circ} \mathrm{C}$ to induce UAS

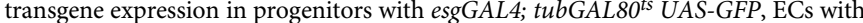
MyolAGAL4; tubGAL80 ${ }^{t s}\left(M y o 1 A^{t s}\right)$, and in VM with how-GAL4; tubGAL0 ${ }^{\text {ts }}$ $\left(h_{0} w^{t s}\right)$. No statistical method was used to predetermine sample size, but typically between 10 and 20 flies were used per experiment. When selecting animals for an experiment, the parental genotype was not concealed since it was required to select relevant progeny. Animals were first selected for their genotype and then randomly chosen to be used for an experiment. See Supplementary Methods for genotypes.

Histology. After fixation in $8 \%$ formaldehyde (+ phosphatase inhibitors: PhosSTOP (Roche)), midguts were washed in PBS, $0.1 \%$ Triton X-100, blocked in PBS, $0.1 \%$ Trinton $\mathrm{X}-100,1 \%$ bovine serum albumin (BSA), $2 \%$ normal goat serum (NGS), and stained in blocking solution with rabbit polyclonal anti-phospho-p38 (Cell Signaling \#9211, 1:50), rabbit polyclonal anti-phospho Ser 10 histone 3 (Upstate Biotechnology/Millipore \#06-570, 1:1000), chicken polyclonal anti- $\beta$ galactosidase (Abcam \#ab9361, 1:1000), and chicken polyclonal anti-GFP (Life Technologies \#A10262, 1:1000). Midguts were then washed in PBS 0.1\% Triton X100 , stained in PBS, $0.3 \%$ Triton X-100, 0.1\% BSA with Alexa Fluor-conjugated secondary antibodies (Life Technologies, 1:1000) and Hoechst 33258 (Life Technologies), and mounted in Vectashield (Vector Laboratories).

Mitotic indices were determined by counting the number of $\mathrm{pH} 3$-positive cells from whole female midguts from two or more independent experiments. The mean number of mitoses per midgut and s.e.m. are presented for each genotype or treatment. Before quantifying the number of mitoses per midgut, the genotype of each sample was concealed. Samples were then randomly analyzed, and the genotype was revealed only after completing analysis.

Statistical analysis. Statistical analyses were performed using GraphPad Prism 5 or 8. For data describing mitoses per midgut, the two-sided Mann-Whitney test (for non-Gaussian data) or the two-sided unpaired $t$ test (for Gaussian data) was applied to determine statistical significance. For qPCR data, the one-way ANOVA test and the Dunnetts sub-test were used on relative mRNA levels not normalized to control. The one sample $t$ test was used on relative mRNA levels normalized to control or treated control. For Stat-GFP or phospho-p38 levels, unpaired $t$ test was used on relative levels not normalized to control. The significance level is indicated by an ${ }^{*}$ for $p \leq 0.05,{ }^{* *}$ for $p \leq 0.01,{ }^{* *}$ for $p \leq 0.001,{ }^{* * * *}$ for $p \leq 0.0001$, and by NS for not significant, $p>0.05$.

Microscopy. Samples were analyzed using Leica M205FA, DM5000B, SP5 and SP8 microscopes. Images were processed with Image $(\mathrm{NIH})$ and Adobe Photoshop CS5. Confocal images are presented as maximal intensity projections of images obtained every $0.5-1.0 \mu \mathrm{m}$. When comparing protein levels, each z-stack was acquired with the same laser intensity and gain, maximal intensity projections of identical dimensions were created and were further similarly processed with Adobe Photoshop CS5. Representative images presented were obtained from $\geq 2$ independent experiments.

Image quantitation. Maximal intensity projections (of 5-6 $\mu$ m thickness) of confocal images from the EC layer of each midgut were generated with ImageJ (NIH). ImageJ was then used to measure the surface area and the signal integrated density for each midgut. The ratio of integrated density:area was determined for each midgut and then normalized to the mean integrated density/area of control midguts to determine the relative signal (phospho-p38 or GFP) levels between treatments and/or genotypes.

Quantitative RT-PCR. RNA was isolated from 20 midguts using an RNAeasy kit (Qiagen) or from 12 midguts using the RNAquous Kit (Ambion). The concentration and quality of the RNA were determined using the Agilent 2200 Tape Station. In all, 250-500 ng of RNA was used for cDNA synthesis using the Quantitect cDNA synthesis kit (Qiagen) or the SuperScript III kit (Invitrogen). qPCR was performed using the LightCycler 480 SYBER Green I Master (Roche), the Maxima SYBER Green/ROX Master (Thermo Fisher Scientific) or the iTaq Universal SYBR Green Supermix (BioRad). SYBR green incorporation during PCR was detected using the Roche LightCycler 480 II, the Applied Biosystems Step One Plus and the CFX384 Touch Real-Time system (BioRad). The following primers were used: upd1 F: 5'-CC ACGTAAGTTTGCATGTTG-3', upd 1 R: 5'-CTAAACAGTAGCCAGGACTC-3', upd2 F: 5'-CACAAGTGCGGTGAAGCTAA-3', upd2 R: 5'-GGCTCTTCTGCTG ATCCTTG-3', upd3 F: 5'-GCCCTCTTCACCAAACTGAA-3', upd3 R: 5'-TTT CTTCTGGATCGCCTTTG-3' from $^{9}$; Socs36E F: $5^{\prime}$-CAGTCAGCAATATGTTG TCG-3', Socs36E R: 5'-ACTTGCAGCATCGTCGCTTC-3' from ${ }^{8}$ and Ask1 F: 5'-TT ACACTCTCAGCTGCGACA-3', Ask1 R: 5'-TGGAGTAGAAGGCCATGGTG-3', GstD1 F: 5' - AGAAGTACGGCAAGACCGAC-3', GstD1 R: 5'-CTTGAAGGCC TCTGGATCGG-3', Mekk1 F: 5'-CGAGTGCTCTCACAGCCATA-3', Mekk1 R: 5'GCCAGTCCTCGACTTTTGAG-3', Tak1 F: 5'-CAGGCAGCCTATGTGGACTT-3', Tak1 R: 5'-GCTTCACCTCCTTCTCGATG-3', lic F: 5'-ACCGCCTTGTAATGGA TTTG-3', lic R: 5'-GCAGATCGTGAAGGAAGAC-3', p38a F:CAGGTGTCAAA GGCAGTTGTT, $p 38$ a R: 5'-GCTCCCGGTACGTCCTCTT-3', $p 38 b$ F: 5-TACGA CCAGAGCTTCGAGGA- $3^{\prime}$ and $p 38 b$ R: 5'-GGCCGGCATTACTGCTCTTT-3'. We found that $\mathrm{crq}$ and $m k 2$ gene expression did not change after $18 \mathrm{~h}$ P.e. infection. In addition, $c r q$ mRNA levels did not change upon all stresses tested (Ecc15, SDS, bleomycin, heat shock, $\mathrm{H}_{2} \mathrm{O}_{2}$, or paraquat). Thus the expression of each target gene was normalized to $\mathrm{crq}$ or $m k 2$ expression (reference) to ensure equivalent RNA or cDNA input. The primers used to detect $c r q$ and $m k 2$ expression were: $c r q$ F: $5^{\prime}-$ CAGAGCTCTCCTCCGAATTG-3', crq R: 5'-ATGCCGGTGATGAGAAAGAC-3', $m k 2$ F: $5^{\prime}$-GGCAAAGGATCTGATCAAGG-3', and $m k 2$ R: 5'-GTTTCCTCGGCC TCCTTTAG-3'. Each assay was performed in triplicate or quadruplet on $\geq 2$ independent biological replicates. All data are presented as relative mRNA levels compared with control (translated from raw Ct values), fold change $=2^{-\Delta \Delta \mathrm{Ct}}$ or $\%$ decrease relative to control with mean and s.e.m.

Reporting summary. Further information on research design is available in the Nature Research Reporting Summary linked to this article.

\section{Data availability}

All data are available from the corresponding authors upon request. The source data underlying Figs. $1 \mathrm{c}-\mathrm{d}, \mathrm{g}, 2 \mathrm{a}-\mathrm{c}, \mathrm{g}, 5 \mathrm{~g}, 6 \mathrm{~d}-\mathrm{f}$, and $7 \mathrm{~d}-\mathrm{e}$ and Supplementary Figs. 1g-h, j, $2 \mathrm{e}-\mathrm{i}, 3 \mathrm{c}, \mathrm{e}, 4 \mathrm{a}-\mathrm{c}, 6 \mathrm{e}, \mathrm{l}-\mathrm{m}, 7 \mathrm{e}, 8 \mathrm{e}, 10 \mathrm{~d}, 11 \mathrm{f}-\mathrm{g}$, and 13 are provided as a Source Data file.

Received: 2 August 2018 Accepted: 28 August 2019

Published online: 25 September 2019 


\section{References}

1. Buchon, N., Silverman, N. \& Cherry, S. Immunity in Drosophila melanogaster-from microbial recognition to whole-organism physiology. Nat. Rev. Immunol. 14, 796-810 (2014).

2. Ohlstein, B. \& Spradling, A. The adult Drosophila posterior midgut is maintained by pluripotent stem cells. Nature 439, 470-474 (2006).

3. Micchelli, C. A. \& Perrimon, N. Evidence that stem cells reside in the adult Drosophila midgut epithelium. Nature 439, 475-479 (2006).

4. Sahai-Hernandez, P., Castanieto, A. \& Nystul, T. G. Drosophila models of epithelial stem cells and their niches. Wiley Interdiscip. Rev. Dev. Biol. 1, 447-457 (2012).

5. Jiang, H., Tian, A. \& Jiang, J. Intestinal stem cell response to injury: lessons from Drosophila. Cell Mol. Life Sci. 73, 3337-3349 (2016).

6. Miguel-Aliaga, I., Jasper, H. \& Lemaitre, B. Anatomy and physiology of the digestive tract of Drosophila melanogaster. Genetics 210, 357-396 (2018).

7. Karpowicz, P., Perez, J. \& Perrimon, N. The Hippo tumor suppressor pathway regulates intestinal stem cell regeneration. Development 137, 4135-4145 (2010).

8. Jiang, H. et al. Cytokine/Jak/Stat signaling mediates regeneration and homeostasis in the Drosophila midgut. Cell 137, 1343-1355 (2009).

9. Patel, P. H., Dutta, D. \& Edgar, B. A. Niche appropriation by Drosophila intestinal stem cell tumours. Nat. Cell Biol. 17, 1182-1192 (2015).

10. Buchon, N., Broderick, N. A., Chakrabarti, S. \& Lemaitre, B. Invasive and indigenous microbiota impact intestinal stem cell activity through multiple pathways in Drosophila. Genes Dev. 23, 2333-2344 (2009).

11. Shaw, R. L. et al. The Hippo pathway regulates intestinal stem cell proliferation during Drosophila adult midgut regeneration. Development 137, 4147-4158 (2010)

12. Staley, B. K. \& Irvine, K. D. Warts and Yorkie mediate intestinal regeneration by influencing stem cell proliferation. Curr. Biol. 20, 1580-1587 (2010).

13. Roux, P. P. \& Blenis, J. ERK and p38 MAPK-activated protein kinases: a family of protein kinases with diverse biological functions. Microbiol Mol. Biol. Rev. 68, 320-344 (2004).

14. Biteau, B., Hochmuth, C. E. \& Jasper, H. JNK activity in somatic stem cells causes loss of tissue homeostasis in the aging Drosophila gut. Cell Stem Cell 3, 442-455 (2008).

15. Beebe, K., Lee, W. C. \& Micchelli, C. A. JAK/STAT signaling coordinates stem cell proliferation and multilineage differentiation in the Drosophila intestinal stem cell lineage. Dev. Biol. 338, 28-37 (2010)

16. Osman, D. et al. Autocrine and paracrine unpaired signaling regulate intestinal stem cell maintenance and division. J. Cell Sci. 125, 5944-5949 (2012).

17. Zhou, F., Rasmussen, A., Lee, S. \& Agaisse, H. The UPD3 cytokine couples environmental challenge and intestinal stem cell division through modulation of JAK/STAT signaling in the stem cell microenvironment. Dev. Biol. 373, 383-393 (2013).

18. Karin, M. \& Clevers, H. Reparative inflammation takes charge of tissue regeneration. Nature 529, 307-315 (2016).

19. Houtz, P. et al. Hippo, TGF-beta, and Src-MAPK pathways regulate transcription of the upd3 cytokine in Drosophila enterocytes upon bacterial infection. PLoS Genet. 13, e1007091 (2017).

20. Kim, D. H. et al. A conserved p38 MAP kinase pathway in Caenorhabditis elegans innate immunity. Science 297, 623-626 (2002).

21. Ha, E. M. et al. Coordination of multiple dual oxidase-regulatory pathways in responses to commensal and infectious microbes in Drosophila gut. Nat. Immunol. 10, 949-957 (2009).

22. Ha, E. M., Oh, C. T., Bae, Y. S. \& Lee, W. J. A direct role for dual oxidase in Drosophila gut immunity. Science 310, 847-850 (2005).

23. Chakrabarti, S., Poidevin, M. \& Lemaitre, B. The Drosophila MAPK p38c regulates oxidative stress and lipid homeostasis in the intestine. PLoS Genet 10, e1004659 (2014).

24. Gupta, J. et al. Dual function of p38alpha MAPK in colon cancer: suppression of colitis-associated tumor initiation but requirement for cancer cell survival. Cancer Cell 25, 484-500 (2014).

25. Otsuka, M. et al. Distinct effects of p38alpha deletion in myeloid lineage and gut epithelia in mouse models of inflammatory bowel disease. Gastroenterology 138, 1255-1265 (2010). 1265 e1251-1259.

26. Jones, R. M. et al. Symbiotic lactobacilli stimulate gut epithelial proliferation via Nox-mediated generation of reactive oxygen species. EMBO J. $\mathbf{3 2}$ 3017-3028 (2013).

27. Kato, M. et al. The ROS-generating oxidase Nox1 is required for epithelial restitution following colitis. Exp. Anim. 65, 197-205 (2016).

28. Cuadrado, A. \& Nebreda, A. R. Mechanisms and functions of p38 MAPK signalling. Biochem J. 429, 403-417 (2010).

29. Chen, J. et al. Participation of the p38 pathway in Drosophila host defense against pathogenic bacteria and fungi. Proc. Natl Acad. Sci. USA 107, 20774-20779 (2010).
30. Brun, S. et al. The MAPKKK Mekk1 regulates the expression of Turandot stress genes in response to septic injury in Drosophila. Genes Cells 11, 397-407 (2006).

31. Vidal, S. et al. Mutations in the Drosophila dTAK1 gene reveal a conserved function for MAPKKKs in the control of rel/NF-kappaB-dependent innate immune responses. Genes Dev. 15, 1900-1912 (2001).

32. Saitoh, M. et al. Mammalian thioredoxin is a direct inhibitor of apoptosis signal-regulating kinase (ASK) 1. EMBO J. 17, 2596-2606 (1998).

33. Santabarbara-Ruiz, P. et al. Ask1 and Akt act synergistically to promote ROSdependent regeneration in Drosophila. PLoS Genet. 15, e1007926 (2019)

34. Santabarbara-Ruiz, P. et al. ROS-Induced JNK and p38 Signaling Is Required for Unpaired Cytokine Activation during Drosophila Regeneration. PLoS Genet. 11, e1005595 (2015)

35. Khan, S. J., Abidi, S. N. F., Skinner, A., Tian, Y. \& Smith-Bolton, R. K. The Drosophila Duox maturation factor is a key component of a positive feedback loop that sustains regeneration signaling. PLoS Genet. 13, e1006937 (2017).

36. Love, N. R. et al. Amputation-induced reactive oxygen species are required for successful Xenopus tadpole tail regeneration. Nat. Cell Biol. 15, 222-228 (2013)

37. Buchon, N. \& Osman, D. All for one and one for all: regionalization of the Drosophila intestine. Insect Biochem Mol. Biol. 67, 2-8 (2015).

38. Belozerov, V. E., Lin, Z. Y., Gingras, A. C., McDermott, J. C. \& Michael Siu, K. W. High-resolution protein interaction map of the Drosophila melanogaster p38 mitogen-activated protein kinases reveals limited functional redundancy. Mol. Cell Biol. 32, 3695-3706 (2012).

39. Shinzawa, N. et al. p38 MAPK-dependent phagocytic encapsulation confers infection tolerance in Drosophila. Cell Host Microbe 6, 244-252 (2009).

40. Seisenbacher, G., Hafen, E. \& Stocker, H. MK2-dependent p38b signalling protects Drosophila hindgut enterocytes against JNK-induced apoptosis under chronic stress. PLoS Genet. 7, e1002168 (2011).

41. Seong, K. H., Li, D., Shimizu, H., Nakamura, R. \& Ishii, S. Inheritance of stress-induced, ATF-2-dependent epigenetic change. Cell 145, 1049-1061 (2011).

42. Kuranaga, E. et al. Reaper-mediated inhibition of DIAP1-induced DTRAF1 degradation results in activation of JNK in Drosophila. Nat. Cell Biol. 4, 705-710 (2002).

43. Ma, Q. Role of nrf2 in oxidative stress and toxicity. Annu Rev. Pharm. Toxicol. 53, 401-426 (2013)

44. Patel, P. H., Maldera, J. A. \& Edgar, B. A. Stimulating cROSstalk between commensal bacteria and intestinal stem cells. EMBO J. 32, 3009-3010 (2013)

45. Hervera, A. et al. Reactive oxygen species regulate axonal regeneration through the release of exosomal NADPH oxidase 2 complexes into injured axons. Nat. Cell Biol. 20, 307-319 (2018).

46. Huang, D. et al. Regulation of Hippo signalling by p38 signalling. J. Mol. Cell Biol. 8, 328-337 (2016)

47. Tobiume, K., Saitoh, M. \& Ichijo, H. Activation of apoptosis signal-regulating kinase 1 by the stress-induced activating phosphorylation of pre-formed oligomer. J. Cell Physiol. 191, 95-104 (2002).

48. Nadeau, P. J., Charette, S. J., Toledano, M. B. \& Landry, J. Disulfide bondmediated multimerization of Ask1 and its reduction by thioredoxin-1 regulate $\mathrm{H}(2) \mathrm{O}(2)$-induced c-Jun $\mathrm{NH}(2)$-terminal kinase activation and apoptosis. Mol. Biol. Cell 18, 3903-3913 (2007).

49. Sekine, Y. et al. The Kelch repeat protein KLHDC10 regulates oxidative stress induced ASK1 activation by suppressing PP5. Mol. Cell 48, 692-704 (2012).

50. Dutta, D. et al. Regional cell-specific transcriptome mapping reveals regulatory complexity in the adult Drosophila midgut. Cell Rep. 12, 346-358 (2015).

51. Xu, C., Luo, J., He, L., Montell, C. \& Perrimon, N. Oxidative stress induces stem cell proliferation via TRPA1/RyR-mediated $\mathrm{Ca}(2+)$ signaling in the Drosophila midgut. eLife 6, e22441 (2017).

52. West, C. \& Silverman, N. p38b and JAK-STAT signaling protect against Invertebrate iridescent virus 6 infection in Drosophila. PLoS Pathog. 14, e1007020 (2018).

53. Srinivasan, N. et al. Actin is an evolutionarily-conserved damage-associated molecular pattern that signals tissue injury in Drosophila melanogaster. eLife 5, e19662 (2016).

54. Wakeman, D. et al. Deletion of p38-alpha mitogen-activated protein kinase within the intestinal epithelium promotes colon tumorigenesis. Surgery 152 286-293 (2012).

55. Biteau, B. et al. Lifespan extension by preserving proliferative homeostasis in Drosophila. PLoS Genet. 6, e1001159 (2010)

56. Goulas, S., Conder, R. \& Knoblich, J. A. The Par complex and integrins direct asymmetric cell division in adult intestinal stem cells. Cell Stem Cell $\mathbf{1 1}$ 529-540 (2012).

57. Adachi-Yamada, T. et al. p38 mitogen-activated protein kinase can be involved in transforming growth factor beta superfamily signal transduction in Drosophila wing morphogenesis. Mol. Cell Biol. 19, 2322-2329 (1999).

58. Sykiotis, G. P. \& Bohmann, D. Keap1/Nrf2 signaling regulates oxidative stress tolerance and lifespan in Drosophila. Dev. Cell 14, 76-85 (2008). 


\section{Acknowledgements}

We thank the Bloomington Stock Center, Vienna Drosophila RNAi Center, Kyoto Stock Center, Zurich ORFeome Project (FlyORF), Developmental Studies Hybridoma Bank, Dirk Bohmann, Shunsuke Ishii, Masayaki Miura, Florenci Serras, and Hugo Stocker for fly stocks and antibodies. This work was financially supported by ERC Advanced Grant 268515, DFG SFB 873, the Huntsman Cancer Foundation, NIH RO1 GM124434 (B.A.E), Helmholtz Zukunftsthema Aging and Metabolic Programming (AMPro) (A.A.T.), and by the Elizabeth Blackwell Institute for Health Research, University of Bristol and the Wellcome Trust Institutional Strategic Support Fund (204813/Z/16/Z) (P.H.P.).

\section{Author contributions}

The project was conceived and developed by P.H.P. and B.A.E. P.H.P. contributed Figs. 1a-c, e-j, 2c-g, 3c-f, 4a-f, 5a-k, 6a-d, 7a-c, f-h, 8, and Supplementary Figs. 1a-d, 1h-j, 3, 4a, c, d, 5c-f, 6a-e, 7, 8a-d, f-i, 9c-j, 10, 11a-e, 12; C.P. contributed 2a, 6e, f, 7d, e and Supplementary Fig. 11f, g; M.K. contributed Fig. 3a, b and Supplementary Figs. 5a, b, g, h, $6 \mathrm{f}-\mathrm{k}, 9 \mathrm{a}, \mathrm{b}$ and contributed to Figs. $4 \mathrm{a}-\mathrm{c}, 5 \mathrm{~d}-\mathrm{f}$ and Supplementary Fig. 8a,d; M.R. contributed Figs. 1d, 2b and Supplementary Fig. 1j, 2a-d, e-g, 13 and contributed to Figs. 1a, b, 3c, d and Supplementary Fig. 1a, b; B.P. contributed Supplementary Figs. 1e-g, 4b, $6 \mathrm{l}$ and contributed to Fig. 7a-c and Supplementary Figs. 11a-e; A.T. contributed Fig. 5g and Supplementary Fig. 2e, h, i, 6m, 8e. P.H.P. wrote and prepared the paper. B.A.E., A.A.T. and P.H.P. edited the paper.

\section{Additional information}

Supplementary Information accompanies this paper at https://doi.org/10.1038/s41467019-12336-w.
Competing interests: The authors declare no competing interests.

Reprints and permission information is available online at http://npg.nature.com/ reprintsandpermissions/

Peer review information Nature Communications thanks the anonymous reviewers for their contribution to the peer review of this work.

Publisher's note Springer Nature remains neutral with regard to jurisdictional claims in published maps and institutional affiliations.

(c) Open Access This article is licensed under a Creative Commons Attribution 4.0 International License, which permits use, sharing, adaptation, distribution and reproduction in any medium or format, as long as you give appropriate credit to the original author(s) and the source, provide a link to the Creative Commons license, and indicate if changes were made. The images or other third party material in this article are included in the article's Creative Commons license, unless indicated otherwise in a credit line to the material. If material is not included in the article's Creative Commons license and your intended use is not permitted by statutory regulation or exceeds the permitted use, you will need to obtain permission directly from the copyright holder. To view a copy of this license, visit http://creativecommons.org/ licenses/by/4.0/.

(C) The Author(s) 2019 